\title{
Traces for Coalgebraic Components
}

\author{
ICHIRO HASUO ${ }^{1,3}$ and BART JACOBS ${ }^{2}$ \\ ${ }^{1}$ Research Institute for Mathematical Sciences, Kyoto University, Kyoto 606-8502, Japan \\ ${ }^{2}$ Institute for Computing and Information Sciences, Radboud University Nijmegen, \\ P.O.Box 9010, 6500 GL Nijmegen, the Netherlands \\ 3 PRESTO Research Promotion Program, Japan Science and Technology Agency
}

Received September 18, 2010

This paper contributes a feedback operator, in the form of a monoidal trace, to the theory of coalgebraic, state-based modelling of components. The feedback operator on components is shown to satisfy the trace axioms of Joyal, Street and Verity. We employ McCurdy's tube diagrams, an extension of standard string diagrams for monoidal categories, for representing and manipulating component diagrams. The microcosm principle then yields a canonical "inner" traced monoidal structure on the category of resumptions (elements of final coalgebras / components). This generalises an observation by Abramsky, Haghverdi and Scott.

\section{Introduction}

The subject of study in the field of coalgebra is state-based computation. A computer is a device that has an internal state, roughly given by the content of all its memory cells and registers, that is not directly observable. However, a user can observe and modify part of this state via I/O devices, such as a screen or keyboard. Very abstractly, such a computer is captured as a coalgebra $X \rightarrow F(X)$, where $X$ represents the state, and $F$ captures the type of operations (for observation and modification) that one can perform on these states. A simple example is a deterministic automaton of the form $X \rightarrow(X \times B)^{A}$ where $A$ is a type for input, and $B$ for output.

The coalgebraic view on state-based systems yields a generic view, for instance, on bisimilarity (indistinguishability of states) and compositionality (see e.g. (Turi and Plotkin, 1997)), and on modal logic (see e.g. (Kurz and Pattinson, 2005)), giving a way to reason about properties of states with dynamic operators like 'nexttime'. Here we use coalgebras to (further) develop a calculus of components, which describes various ways of combining components (smaller subsystems) into larger systems. Numerous component calculi have been proposed for the purpose of aiding modular design of complex systems, such as Reo (Arbab, 2004). The existing component calculi come with different sets of (typically several) component connectors. In earlier work (Hasuo et al., 2009; Asada and Hasuo, 2010) we have focused on the very core subset of such calculi, with sequential composition 
and parallel composition only. In this paper we add a feedback operator to this calculus, in the form of a trace.

This calculus may be understood as a many-sorted process algebra that acts directly on systems; by employing the microcosm principle we obtain process algebraic operators on behaviours, too, see (Hasuo et al., 2008; Hasuo et al., 2009).

A categorical approach to system composition has been developed in (Katis et al., 1997) based on bicategories. The bicategorical aspect comes up if one uses components as morphisms, because composition of components is associative only up to isomorphism, see Lemma 4.2.3 below. This approach is extended in (Barbosa, 2001; Barbosa, 2003) with monads, to allow for different kinds of computation (Moggi, 1991). Here, like in (Hasuo et al., 2008; Hasuo et al., 2009) we take a slightly different approach and use components as objects in a category, with fixed input and output. In order to deal with relabelling of input and output, we need to organise the whole as an indexed category. This indexing however is straightforward and poses no technical obstacles.

A crucial ingredient that is missing so far in these calculi of components is feedback. It makes it possible to include "loops" in diagrams of components, in order to capture recursive flows. Here we extend coalgebraic component calculi with such a feedback mechanism, in the form of a trace operator. Traces as feedback operators have been introduced abstractly in (Joyal et al., 1996). Here we essentially follow this framework, but the required identities for these traces only hold up to isomorphism (just like for composition). The traces that we introduce for coalgebraic components are based on the trace construction in Kleisli categories from (Jacobs, 2010). In his thesis Barbosa discusses a "partial feedback" operator (see (Barbosa, 2001, Ch..,$\S 51)$ ) but it is not a proper trace operator (in the sense of (Joyal et al., 1996)) because it does not have the right type, nor the right behaviour.

For components it is very useful to have a diagrammatic language that makes it possible to build a composite system in a picture with lines representing connections between them. There is a standard language of string diagrams for monoidal categories, see (Penrose, 1971; Joyal and Street, 1991). Here we employ McCurdy's extension (McCurdy, 2010) - which we call tube diagrams - for capturing coalgebraic components and their connections. This language can be used to reason about coalgebraic components, via specific diagrammatic manipulations, see Section 5. The additional (third) dimensionobtained by using tubes rather than strings - is needed because in our calculus input can be structured both multiplicatively (with tensor $\otimes$ ) and additively (with coproduct + ). The feedback/trace operator works with respect to this additive structure.

The additional trace operator for components embodies iteration in data processing - a fundamental concept in computer science. Our theory is generic because it is parametrised by a monad $T$ : the monad represents the computational effect that makes an iterated function "total". The prototypical example of such effect is partiality (i.e. $T=1+(-)$, the lift monad) but non-determinism and probability also fits in. To demonstrate the versatility of our results, we derive the traced monoidal structure of the category of T-resumptions, in a canonical manner. This generalises the observation in (Abramsky et al., 2002): there the authors focus on the partiality effect (i.e. $T=1+(-)$ ) and the trace operator on resumptions is introduced in concrete terms. Here we, instead, 
derive trace operators on various resumptions uniformly from the trace operators on components, in the style of (Krstić et al., 2001). The derived traced monoidal category lives in the "traced monoidal bicategory" of components, providing an instance of the microcosm principle (Baez and Dolan, 1998; Hasuo et al., 2008; Hasuo et al., 2009). Another way to look at our construction is that it uniformly transforms the traced monoidal category of $T$-computations (namely the Kleisli category $\mathcal{K}(T)$ ) into the traced monoidal category of $T$-strategies (identified with $T$-resumptions). By further applying the Int-construction (Joyal et al., 1996), one obtains the compact closed category of (stateful) T-games, as pointed out in (Abramsky and Jagadeesan, 1994).

The paper is organised as follows. After some preliminary material it starts in Section 3 with the fundamental operator of 'state extension' that adds a state object, either on the left or on the right, to a coalgebraic component. It will play an important role in the rest of the paper, for instance in Section 4 on the various composition operators: sequential $\gg$, multiplicative parallel || and additive parallel $\square$. Subsequently, Section 5 describes the tube calculus, with several distributivity results. Section 6 defines traces for components and proves that the trace axioms hold; this is our main contribution. Finally in Section 7 we identify the category of resumptions as an "inner" traced monoidal category in that of components.

\section{Preliminaries}

The basic setting is described by a category $\mathbb{C}$ with some structure and a monad $T$ on $\mathbb{C}$. We assume in the first place that $\mathbb{C}$ is a symmetric monoidal category with tensor $\otimes: \mathbb{C} \times \mathbb{C} \rightarrow \mathbb{C}$ and tensor unit $I \in \mathbb{C}$, together with canonical isomorphisms:

$$
X \otimes(Y \otimes Z) \stackrel{\alpha}{\cong}(X \otimes Y) \otimes Z \quad I \otimes X \stackrel{\lambda}{\cong} X \quad X \otimes Y \stackrel{\gamma}{\stackrel{\gamma}{\longrightarrow} Y \otimes X}
$$

One often writes $\rho=\lambda \circ \gamma: X \otimes I \stackrel{\cong}{\longrightarrow} X$.

We assume that the monad $T=(T, \eta, \mu)$ on $\mathbb{C}$ is symmetric monoidal (also known as commutative), via a natural transformation with components dst: $T(X) \otimes T(Y) \rightarrow$ $T(X \otimes Y)$ interacting appropriately with the monoidal isomorphisms (1) and with the unit $\eta$ and multiplication $\mu$ of the monad. For this natural transformation dst we write st $=$ dst $\circ(\eta \otimes \mathrm{id}): X \otimes T(Y) \rightarrow T(X \otimes Y)$ for the 'strength' map of the monad. The abbreviation 'dst' stands for 'double strength'.

The Kleisli category of the monad $T$ is written as $\mathcal{K} \ell(T)$. Usually we write a fat dot for composition in $\mathcal{K} \ell(T)$, if we wish to distinguish it from ordinary composition $\circ$ in $\mathbb{C}$. The inclusion functor $J: \mathbb{C} \rightarrow \mathcal{K} \ell(T)$, given by $X \mapsto X$ and $f \mapsto \eta \circ f$ is sometimes not written explicitly, when context allows so.

We also assume that the category $\mathbb{C}$ has (distributive) coproducts,+ 0 , written also as $\amalg$ when indexed over a set. The associated coprojections will be written as $\kappa_{i}: X_{i} \rightarrow$ $X_{1}+X_{2}$, and cotupling of $f_{i}: X_{i} \rightarrow Y$ is written as $\left[f_{1}, f_{2}\right]: X_{1}+X_{2} \rightarrow Y$. Distributivity here means that the tensor $\otimes$ distributes over coproducts $(0,+)$. In the binary case this 
means that the canonical maps:

$$
\operatorname{dis} \stackrel{\text { def }}{=}\left(X \otimes A+X \otimes B \stackrel{\left[\operatorname{id}_{X} \otimes \kappa_{1}, \operatorname{id}_{X} \otimes \kappa_{2}\right]}{\longrightarrow} X \otimes(A+B)\right)
$$

are isomorphisms. Additionally, the canonical map $0 \rightarrow X \otimes 0$ is an isomorphism; this is the nullary case of distributivity. Notice that if the category $\mathbb{C}$ is monoidal closed, these isomorphisms automatically exist, since $X \otimes-$ is then left adjoint to exponentiation $X \multimap(-)$.

We list some elementary equations for this distribution map dis that will be used later. The proofs are easy and left to the reader.

Lemma 2.1. The distribution map dis defined in (2) satisfies

1 naturality: dis $\circ(f \otimes g+f \otimes h)=(f \otimes(g+h)) \circ$ dis;

$2 \alpha \circ(\mathrm{id} \otimes$ dis $) \circ$ dis $=$ dis $\circ(\alpha+\alpha)$;

$3 \lambda \circ$ dis $=\lambda+\lambda$.

There are also rules regulating the interaction of this distribution map (2) with the monoidal isomorphisms associated with coproducts. We shall label them with a + , as in $\alpha_{+}$, in order to distinguish them from the isomorphisms for $\otimes$.

Lemma 2.2. The distributivity map dis interacts with the monoidal isomorphisms for + as:

1 Interaction with $\rho_{+}$:

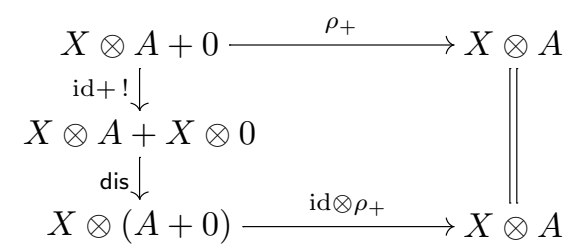

2 Interaction with $\gamma_{+}$:

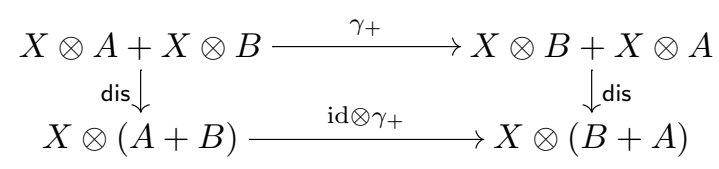

3 Interaction with $\alpha_{+}$:

$$
\begin{aligned}
& (X \otimes A)+((X \otimes C)+(X \otimes D)) \stackrel{\alpha_{+}}{\longrightarrow}((X \otimes A)+(X \otimes C))+(X \otimes D) \\
& \text { id }+ \text { dis } \downarrow \\
& (X \otimes A)+(X \otimes(C+D)) \\
& \downarrow \text { dis }+ \text { id } \\
& \operatorname{dis} \downarrow \quad \operatorname{id} \otimes \alpha_{+} \quad \downarrow \text { dis } \\
& \left.X \otimes(A+(C+D)) \longrightarrow X \otimes\left(\text { id } \otimes \alpha_{+} \longrightarrow C+C\right)+D\right)
\end{aligned}
$$




\section{Coalgebraic components and state extension}

This section describes the basic construction of what we call 'state extension' for coalgebraic components. It serves as an auxiliary operator for the constructions like composition and trace in later sections. Formally, state extension is described as an action of a (monoidal) category on a category of components, see (Janelidze and Kelly, 2001). This insight is not important for what follows, so it is elaborated separately in Section 3.1.

Like in (Barbosa, 2001; Barbosa, 2003; Hasuo et al., 2009) we consider coalgebraic components of the form:

$$
X \otimes A \stackrel{c}{\longrightarrow} T(X \otimes B)
$$

where $X$ is the state space, $A$ the type/object of inputs and $B$ the type of outputs. In general we shall use letters like $X, Y, Z, U, V$ for states and $A, B, C$ for in/outputs. The monad $T$ captures the type of computation involved, following the standard approach in monadic computation. For instance, non-deterministic for $T=$ powerset, partial for $T=\operatorname{lift}$, probabilistic for $T=$ distribution, with side-effects for $T=(S \times-)^{S}$ with $S$ for states, or even deterministic for $T=$ identity. Throughout this article $T$ will be used as parameter. It will be made explicit when certain additional requirements are needed. Often this $T$ is invisible, when we are working in the Kleisli category $\mathcal{K} \ell(T)$ of $T$.

Strictly speaking the map $c$ in $(3)$ is not a coalgebra. In case the category $\mathbb{C}$ is closed, with $\multimap$ as exponent (internal hom), this map $c$ may equivalently be written in coalgebra form $X \longrightarrow A \multimap T(X \otimes B)$. By using the form (3) we can work without the closedness assumption on the category $\mathbb{C}$.

For fixed objects $A, B \in \mathbb{C}$ we thus have a category of such coalgebraic components which we shall write as $\operatorname{Comp}(T, A, B)$. A morphism

$$
(X \otimes A \stackrel{c}{\longrightarrow} T(X \otimes B)) \stackrel{f}{\longrightarrow}(Y \otimes A \stackrel{d}{\longrightarrow} T(Y \otimes B))
$$

in $\operatorname{Comp}(T, A, B)$ is a map $f: X \rightarrow Y$ in $\mathbb{C}$ satisfying $T\left(f \otimes \operatorname{id}_{B}\right) \circ c=d \circ\left(f \otimes \operatorname{id}_{A}\right)$. There is thus an obvious forgetful functor $\operatorname{Comp}(T, A, B) \rightarrow \mathbb{C}$ that maps a coalgebraic component to its underlying state.

Each "pure" map $f: A \rightarrow B$ in $\mathbb{C}$ gives rise to a coalgebraic component written as arr $f$ in $\operatorname{Comp}(T, A, B)$, with the tensor unit $I$ as trivial state space, as in:

$$
\operatorname{arr} f \stackrel{\text { def }}{=}\left(I \otimes A \stackrel{\operatorname{id}_{I} \otimes f}{\longrightarrow} I \otimes B \stackrel{\eta}{\longrightarrow} T(I \otimes B)\right) .
$$

For maps $g: C \rightarrow A$ and $h: B \rightarrow D$ in $\mathbb{C}$ there is an obvious relabelling functor $(g, h)^{*}: \operatorname{Comp}(T, A, B) \rightarrow \operatorname{Comp}(T, C, D)$ given by:

$$
(X \otimes A \stackrel{c}{\longrightarrow} T(X \otimes B)) \longmapsto(X \otimes C \stackrel{\mathrm{id} \otimes g}{\longrightarrow} X \otimes A \stackrel{c}{\longrightarrow} T(X \otimes B) \stackrel{T(\mathrm{id} \otimes h)}{\longrightarrow} T(X \otimes D)) .
$$

On morphisms of coalgebraic components relabelling is the identity. Components thus form a functor $\operatorname{Comp}(T,-,-): \mathbb{C}^{\mathrm{op}} \times \mathbb{C} \rightarrow$ Cat. They can be described as Cat-valued distributors/profunctors/arrows, as shown in (Hasuo et al., 2008; Hasuo et al., 2009; Asada and Hasuo, 2010).

With these definitions in place we can introduce state extension. 
Definition 3.1. For a coalgebraic component $X \otimes A \stackrel{c}{\rightarrow} T(X \otimes B)$ with state space $X$, as in (3), and for objects $U, V \in \mathbb{C}$ we define two new coalgebraic components $U \mid c$ and $c \mid V$, with extended state spaces $U \otimes X$ and $X \otimes V$ respectively, namely:

$$
\begin{array}{cc}
(U \otimes X) \otimes A \longrightarrow c & T((U \otimes X) \otimes B) \\
\alpha^{-1} \downarrow \cong & \cong \uparrow T(\alpha) \\
U \otimes(X \otimes A) \stackrel{\mathrm{id} \otimes c}{\longrightarrow} U \otimes T(X \otimes B) \stackrel{\text { st }}{\longrightarrow} T(U \otimes(X \otimes B)) \\
(X \otimes V) \otimes A \stackrel{c \mid V}{\longrightarrow} T((X \otimes V) \otimes B) \\
\alpha^{-1} \circ(\gamma \otimes \mathrm{id}) \downarrow \cong \\
V \otimes(X \otimes A) \stackrel{\mathrm{id} \otimes c}{\longrightarrow} V \otimes T(X \otimes B) \stackrel{\text { st }}{\longrightarrow} T(V \otimes(X \otimes B))
\end{array}
$$

Clearly, $c \mid V=T(\gamma \otimes$ id $) \circ(V \mid c) \circ(\gamma \otimes$ id $)$.

State extension can be defined more conveniently directly in the Kleisli category $\mathcal{K} \ell(T)$, namely as $U \mid c=\alpha \bullet(U \otimes c) \bullet \alpha^{-1}$, and $d \mid V=(\gamma \otimes$ id $) \bullet(V \mid d) \bullet(\gamma \otimes$ id $)$. Also reasoning about these constructions is easier when done directly in the Kleisli category. In the proof of the main lemma below we shall use a mix between reasoning in $\mathbb{C}$ and in $\mathcal{K} \ell(T)$ in order to demonstrate the difference. Later on in this paper we reason mostly in the Kleisli category.

State extension satisfies some basic properties.

Lemma 3.2. The above operators $U \mid c$ and $c \mid V$ satisfy the following properties.

$1 U \mid \eta=\eta$ and $\eta \mid V=\eta$.

$2 U \mid(\operatorname{arr} f)=\eta \circ\left(\operatorname{id}_{(U \otimes I)} \otimes f\right)$ and $(\operatorname{arr} f) \mid V=\eta \circ\left(\operatorname{id}_{(I \otimes U)} \otimes f\right)$, with arr defined in (5).

$3 \quad$ For two composable components $X \otimes A \stackrel{c}{\rightarrow} T(X \otimes B)$ and $X \otimes B \stackrel{d}{\rightarrow} T(X \otimes C)$ with the same state space, state extension commutes with Kleisli composition $\bullet$, in the sense that:

$$
U \mid(d \bullet c)=(U \mid d) \bullet(U \mid c) \quad \text { and } \quad(d \circ c) \mid V=(d \mid V) \bullet(c \mid V) .
$$

4 State extension with the tensor unit $I$, as trivial state space, is isomorphic to the original component, via maps of components:

$$
\begin{gathered}
T((X \otimes I) \otimes B) \stackrel{T(\rho \otimes \mathrm{id})}{\longrightarrow} T(X \otimes B) \stackrel{T(\lambda \otimes \mathrm{id})}{\cong} T((I \otimes X) \otimes B) \\
c \mid I \uparrow \\
(X \otimes I) \otimes A \stackrel{\rho \otimes \mathrm{id}}{\cong} X \otimes A \stackrel{\uparrow_{c}}{\cong} \underset{\lambda \otimes \mathrm{id}}{\cong}(I \otimes X) \otimes A
\end{gathered}
$$

5 Repeated state extension is isomorphic to tensored state extension:

$$
\begin{gathered}
T((U \otimes(V \otimes X)) \otimes B) \stackrel{T(\alpha \otimes \mathrm{id})}{\cong} T(((U \otimes V) \otimes X) \otimes B) \\
U \mid(V \mid c) \uparrow \\
(U \otimes(V \otimes X)) \otimes A \stackrel{\alpha \otimes \mathrm{id}}{\cong}((U \otimes V) \otimes X) \otimes A
\end{gathered}
$$




$$
\begin{gathered}
T((X \otimes(U \otimes V)) \otimes B) \stackrel{T(\alpha \otimes \mathrm{id})}{\cong} T(((X \otimes U) \otimes V) \otimes B) \\
c \mid(U \otimes V) \uparrow \\
(X \otimes(U \otimes V)) \otimes A \stackrel{\alpha \otimes \mathrm{id}}{\cong}((X \otimes U) \otimes V) \otimes A
\end{gathered}
$$

6 Left and right extension can be exchanged via an isomorphism of components:

$$
\begin{gathered}
T((U \otimes(X \otimes V)) \otimes B) \stackrel{T(\alpha \otimes \mathrm{id})}{\cong} T(((U \otimes X) \otimes V) \otimes B) \\
U \mid(c \mid V) \uparrow \\
(U \otimes(X \otimes V)) \otimes A \stackrel{\alpha \otimes \mathrm{id}}{\cong}((U \otimes X) \otimes V) \otimes A
\end{gathered}
$$

7 For $f: U \rightarrow U^{\prime}$ and $g: V \rightarrow V^{\prime}$ we have:

$$
\begin{aligned}
& \left(U^{\prime} \mid c\right) \circ((f \otimes \mathrm{id}) \otimes \mathrm{id})=T((f \otimes \mathrm{id}) \otimes \mathrm{id}) \circ(U \mid c) \\
& \left(d \mid V^{\prime}\right) \circ((\mathrm{id} \otimes g) \otimes \mathrm{id})=T((\mathrm{id} \otimes g) \otimes \mathrm{id}) \circ(d \mid V) .
\end{aligned}
$$

For a map $h$ in $\mathbb{C}$ between states we have:

$$
\begin{aligned}
T((\mathrm{id} \otimes h) \otimes \mathrm{id}) \circ(U \mid c) & =U \mid(T(h \otimes \mathrm{id}) \circ c) \\
T((h \otimes \mathrm{id}) \otimes \mathrm{id}) \circ(d \mid V) & =(T(h \otimes \mathrm{id}) \circ d) \mid V \\
(U \mid c) \circ((\mathrm{id} \otimes h) \otimes \mathrm{id}) & =U \mid(c \circ(h \otimes \mathrm{id})) \\
(d \mid V) \circ((h \otimes \mathrm{id}) \otimes \mathrm{id}) & =(d \circ(h \otimes \mathrm{id})) \mid V .
\end{aligned}
$$

Consequently, if $h: X \rightarrow Y$ is a homomorphism of coalgebraic components the following diagrams commute.

$$
\begin{aligned}
& T((U \otimes X) \otimes B) \stackrel{T((f \otimes h) \otimes \mathrm{id})}{\longrightarrow} T\left(\left(U^{\prime} \otimes Y\right) \otimes B\right) \\
& U \mid c \uparrow \quad \uparrow_{U^{\prime} \mid d} \\
& (U \otimes X) \otimes A \stackrel{(f \otimes h) \otimes \mathrm{id}}{\longrightarrow}\left(U^{\prime} \otimes Y\right) \otimes A \\
& T((X \otimes V) \otimes B) \stackrel{T((h \otimes g) \otimes \mathrm{id})}{\longrightarrow} T\left(\left(Y \otimes V^{\prime}\right) \otimes B\right) \\
& \begin{array}{c}
c \mid V \uparrow \\
(X \otimes V) \otimes A \stackrel{(h \otimes g) \otimes \mathrm{id}}{\longrightarrow}\left(Y \otimes V^{\prime}\right) \otimes A
\end{array}
\end{aligned}
$$

8 State extension also commutes with relabelling: for $g: C \rightarrow A$ and $h: B \rightarrow D$ we have

$$
(g, h)^{*}(U \mid c)=U \mid\left((g, h)^{*}(c)\right) \text { and }(g, h)^{*}(c \mid V)=\left((g, h)^{*}(c)\right) \mid V .
$$

Proof. Because left and right state extension are related via $c \mid V=T(\gamma \otimes \mathrm{id}) \circ(V \mid$ $c) \circ(\gamma \otimes$ id $)$ we generally only consider one case. For instance for the first point we have:

$$
\begin{aligned}
U \mid \eta & =T(\alpha) \circ \text { st } \circ(\eta \otimes \mathrm{id}) \circ \alpha^{-1} \\
& =T(\alpha) \circ \text { dst } \circ(\eta \otimes \eta) \circ \alpha^{-1}=T(\alpha) \circ \eta \circ \alpha^{-1}=\eta \circ \alpha \circ \alpha^{-1}=\eta .
\end{aligned}
$$

The interaction of state extension and Kleisli composition in the third point of the lemma 
is best shown in the Kleisli category itself:

$$
\begin{aligned}
(U \mid d) \bullet(U \mid c) & =\alpha \bullet(U \otimes d) \bullet \alpha^{-1} \bullet \alpha \bullet(U \otimes c) \bullet \alpha \\
& =\alpha \bullet(U \otimes d) \bullet(U \otimes c) \bullet \alpha \\
& =\alpha \bullet(U \otimes(d \bullet c)) \bullet \alpha \\
& =U \mid(d \bullet c) .
\end{aligned}
$$

The validity of the fourth point follows from:

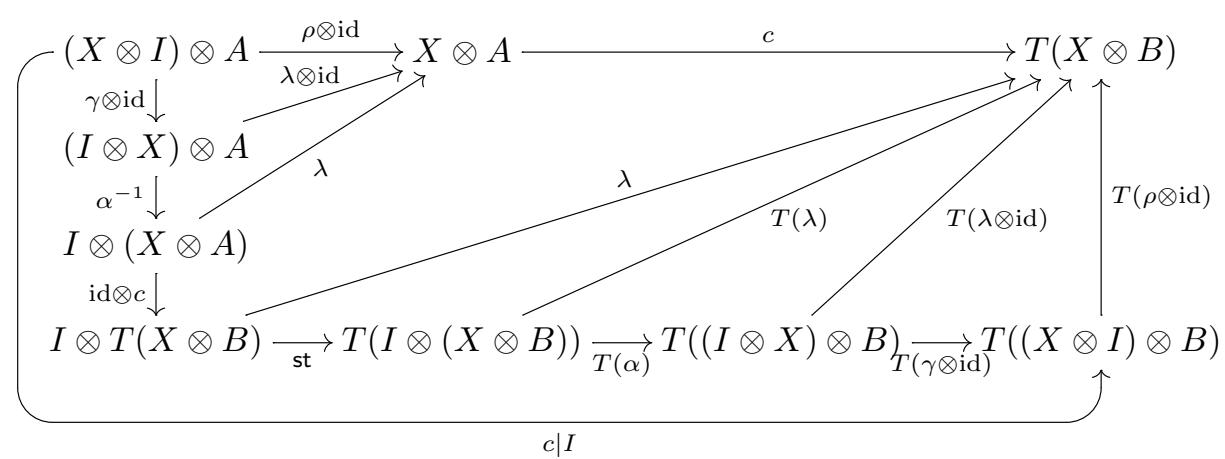

Also for the fifth point we only elaborate one case, in the Kleisli category:

$$
\begin{aligned}
(\alpha & \otimes \mathrm{id}) \bullet(U \mid(V \mid c)) \\
& =(\alpha \otimes \mathrm{id}) \bullet \alpha \bullet\left(U \otimes\left(\alpha \bullet(V \otimes c) \bullet \alpha^{-1}\right)\right) \bullet \alpha^{-1} \\
& =\alpha \bullet \alpha \bullet(U \otimes(V \otimes c)) \bullet\left(U \otimes \alpha^{-1}\right) \bullet \alpha^{-1} \\
& =\alpha \bullet((U \otimes V) \mid c) \bullet \alpha \bullet\left(U \otimes \alpha^{-1}\right) \bullet \alpha^{-1} \\
& =\alpha \bullet((U \otimes V) \mid c) \bullet \alpha^{-1} \bullet(\alpha \otimes \mathrm{id}) \\
& =((U \otimes V) \mid c) \bullet(\alpha \otimes \mathrm{id}) .
\end{aligned}
$$

Verification of the remaining properties proceeds along the same lines, and is left to the interested reader.

\subsection{State extension as action}

The state extension operators | can be described as functors in a the following diagram:

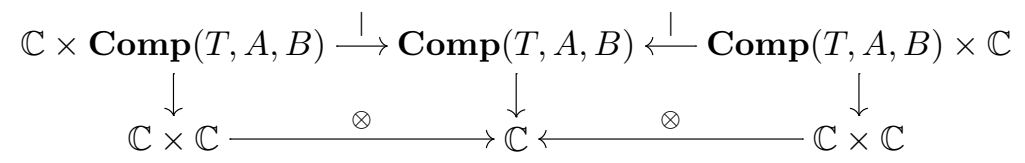

These functors $\mathbb{C} \times \operatorname{Comp}(T, A, B) \rightarrow \operatorname{Comp}(T, A, B)$ and $\operatorname{Comp}(T, A, B) \times \mathbb{C} \rightarrow$ $\operatorname{Comp}(T, A, B)$ at the top this diagram can equivalently be described as (two) functors of the form $\mathbb{C} \rightrightarrows[\operatorname{Comp}(T, A, B), \operatorname{Comp}(T, A, B)]$, from $\mathbb{C}$ to the category of endofunctors on the category $\operatorname{Comp}(T, A, B)$. These two functors are strong monoidal, where the category of endofunctors carries composition and identity (of functors) as a monoidal 
structure. This precisely means that the monoidal category $\mathbb{C}$ acts on $\operatorname{Comp}(T, A, B)$, see (Janelidze and Kelly, 2001), in two ways, namely via left and right state extension.

Moreover, because (left and right) state extensions commute with relabelling they form natural transformations in:

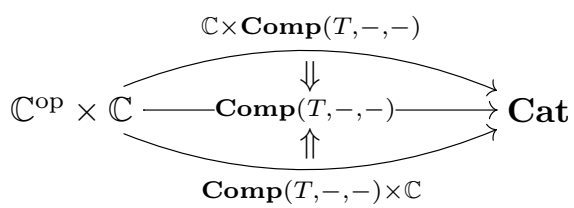

By combining these two observations we conclude that the monoidal category $\mathbb{C}$ acts on the indexed category $\operatorname{Comp}(T,-,-): \mathbb{C}^{\mathrm{op}} \times \mathbb{C} \rightarrow$ Cat, in the sense that there are strong monoidal (left and right) state extension functors:

$$
\mathbb{C} \underset{\text { rse }}{\stackrel{\text { lse }}{\longrightarrow}}[\operatorname{Comp}(T,-,-), \operatorname{Comp}(T,-,-)]
$$

from $\mathbb{C}$ to the category of endomaps on $\operatorname{Comp}(T,-,-)$. Objects of the latter category are natural transformations $\sigma: \operatorname{Comp}(T,-,-) \Rightarrow \operatorname{Comp}(T,-,-)$ and a morphism $M: \sigma \rightarrow \tau$ between such natural transformations is a so-called modification (Kelly and Street, 1974; Jacobs, 1999): a family of natural transformations $M_{(A, B)}: \sigma_{(A, B)} \rightarrow \tau_{(A, B)}$ commuting with relabelling.

We shall elaborate the left state extension case in (6). Each $U \in \mathbb{C}$ yields a natural transformation Ise $(U): \operatorname{Comp}(T,-,-) \rightarrow \operatorname{Comp}(T,-,-)$ between indexed categories, with component on $(A, B) \in \mathbb{C}^{\mathrm{op}} \times \mathbb{C}$ given by the functor Ise $(U)_{(A, B)}: \operatorname{Comp}(T, A, B) \rightarrow$ $\operatorname{Comp}(T, A, B)$, which was written earlier as $c \mapsto U \mid c$. Hence Ise $(U)_{A, B}=(U \mid-)$. This is functorial by Lemma 3.2.7 and natural in $A, B$ by 3.2.(8). Each map $f: U \rightarrow U^{\prime}$ in $\mathbb{C}$ yields a modification Ise $(f): \operatorname{Ise}(U) \rightarrow \operatorname{Ise}\left(U^{\prime}\right)$ between these natural transformations, consisting of a family Ise $(f)_{(A, B)}$ : Ise $(U)_{(A, B)} \rightarrow \operatorname{Ise}\left(U^{\prime}\right)_{(A, B)}$ of natural transformations, with component for a coalgebra $X \otimes A \stackrel{c}{\rightarrow} T(X \otimes B)$ consisting of the map:

$$
\operatorname{Ise}(U)_{(A, B)}(c)=(U \mid c) \underset{\operatorname{|se}(f)_{(A, B), c}}{=f \otimes \operatorname{id}_{X}}\left(U^{\prime} \mid c\right)=\operatorname{Ise}\left(U^{\prime}\right)_{(A, B)}(c) .
$$

We thus have natural transformations:

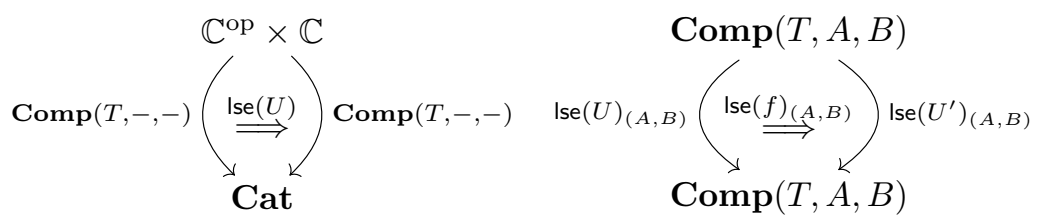

It is not hard to check that Ise $(U)$ is natural (in $A, B$ ) and that these Ise $(f)$ are natural (in $c$ ) and commute with relabelling.

Finally, this functor Ise: $\mathbb{C} \rightarrow[\operatorname{Comp}(T,-,-), \operatorname{Comp}(T,-,-)]$ is strong monoidal 
because it preserves the monoidal structure:

$$
\begin{aligned}
\operatorname{Ise}(I)_{(A, B)}(c) & =I \mid c \\
& \cong c \\
\operatorname{Ise}(U \otimes V)_{(A, B)}(c) & =(U \otimes V) \mid c \\
& \cong U \mid(V \mid c) \\
& =\mid \operatorname{se}(U)_{(A, B)}\left(\operatorname{Ise}(V)_{(A, B)}(c)\right) \\
& =\left(\operatorname{|se}(U)_{(A, B)} \circ \mid \operatorname{se}(V)_{(A, B)}\right)(c),
\end{aligned}
$$

where the category of endomaps $[\operatorname{Comp}(T,-,-), \operatorname{Comp}(T,-,-)]$ carries the standard monoidal structure given by composition as tensor, with identity as tensor unit.

\section{Composition of components}

This section describes three basic forms of composition for coalgebraic components, namely sequential composition $\gg$, multiplicative parallel composition $\|$, and additive parallel composition $\square$. We start with $\gg$, which we can conveniently describe in terms of state extension.

Definition 4.1. The sequential composition operator $\gg$ is defined for coalgebraic components with matching input and output: for

$$
X \otimes A \stackrel{c}{\longrightarrow} T(X \otimes B) \text { and } Y \otimes B \stackrel{d}{\longrightarrow} T(Y \otimes C)
$$

we get $c \gg d$ via composition of Kleisli maps:

$$
c \gg d=((X \otimes Y) \otimes A \stackrel{c \mid Y}{\longrightarrow}(X \otimes Y) \otimes B \stackrel{X \mid d}{\longrightarrow}(X \otimes Y) \otimes C) .
$$

Thus $c \gg d$ involves first doing $c$ and then $d$, on a combined state space $X \otimes Y$.

The notation $\gg$ for composition is as used for arrows, see e.g. (Jacobs et al., 2009). Composition of components satisfies the properties of composition for arrows, but only up to (canonical) isomorphisms. This will be shown next.

Lemma 4.2. The following equations and isomorphisms (of coalgebraic components) hold for sequential composition.

$1 \operatorname{arr}(f) \gg \operatorname{arr}(g)=(\operatorname{arr}(g \circ f) \mid I)=(I \mid \operatorname{arr}(g \circ f)) \stackrel{\lambda=\rho}{\cong} \operatorname{arr}(g \circ f)$ in the following isomorphism of coalgebraic components.

$$
\begin{aligned}
& T((I \otimes I) \otimes C) \stackrel{T(\lambda \otimes \mathrm{id})=T(\rho \otimes \mathrm{id})}{\cong} T(I \otimes C) \\
& \operatorname{arr}(f) \ggg \operatorname{arr}(g)=(\operatorname{arr}(g \circ f) \mid I)=(I \mid \operatorname{arr}(g \circ f)) \uparrow \quad \cong \quad \cong \operatorname{arr}(g \circ f) \\
& (I \otimes I) \otimes A \underset{(\lambda \otimes \mathrm{id})=(\rho \otimes \mathrm{id})}{\cong} I \otimes A
\end{aligned}
$$


$2 \quad c \gg \operatorname{arr}(g) \stackrel{\rho}{\cong} T(\mathrm{id} \otimes g) \circ c$ and $\operatorname{arr}(f) \gg d \underset{\cong}{\stackrel{\lambda}{\cong}} d \circ(\mathrm{id} \otimes f)$ in:

$$
\begin{aligned}
& T((X \otimes I) \otimes C) \stackrel{T(\rho \otimes \mathrm{id})}{\cong} T(X \otimes C) \quad T((I \otimes Y) \otimes C) \stackrel{T(\lambda \otimes \mathrm{id})}{\cong} T(Y \otimes C) \\
& c \ggg \operatorname{arr}(g) \uparrow \quad \cong \quad T(\mathrm{id} \hat{\otimes} g) \circ c \quad \operatorname{arr}(\hat{f} \hat{)} \gg d \quad \cong \quad \cong d o(\operatorname{id} \otimes f) \\
& (X \otimes I) \otimes A \underset{\rho \otimes \text { id }}{\stackrel{\cong}{\cong}} X \otimes A \quad(I \otimes Y) \otimes A \underset{\lambda \otimes \text { id }}{\stackrel{\cong}{\cong}} Y \otimes A
\end{aligned}
$$

In particular, arr(id) is unit for $\gg$, up-to-isomorphism.

$3 \quad(c \gg(d \gg e)) \stackrel{\alpha}{\cong}((c \gg d) \ggg e)$, in:

$$
\begin{aligned}
& T((X \otimes(Y \otimes Z)) \otimes D) \stackrel{T(\alpha \otimes \mathrm{id})}{\cong} T(((X \otimes Y) \otimes Z) \otimes D) \\
& (c \gg(d \gg e) \uparrow \\
& \quad(X \otimes(Y \otimes Z)) \otimes A \underset{\uparrow}{\cong} \underset{\alpha \otimes \text { id }}{\longrightarrow}((X \otimes Y) \otimes Z) \otimes A
\end{aligned}
$$

4 For appropriately typed maps in $\mathbb{C}$ between states:

$$
\begin{aligned}
& ((f \otimes g) \otimes \mathrm{id}) \bullet(c \gg d)=((f \otimes \mathrm{id}) \bullet c) \ggg((g \otimes \mathrm{id}) \bullet d) \\
& (c \gg d) \bullet((f \otimes g) \otimes \mathrm{id})=(c \bullet(f \otimes \mathrm{id})) \gg(d \bullet(g \otimes \mathrm{id}))
\end{aligned}
$$

As a result, sequential composition $\gg$ of components is a functor of the form $\operatorname{Comp}(T, A, B) \times \operatorname{Comp}(T, B, C) \rightarrow \operatorname{Comp}(T, A, C)$.

The last point suggests the notation used in (Hasuo et al., 2009) for the type of $\gg$, namely $(A, B) \times(B, C) \rightarrow(A, C)$. We shall also use it later on, especially in Section 6 .

Proof. All this follows from Lemma 3.2. The numbers labelling the equations below refer to the items in this lemma. Recall that we use $\bullet$ for composition in the Kleisli category of the monad $T$ and $\circ$ for composition in $\mathbb{C}$.

1 Since:

$$
\begin{aligned}
\operatorname{arr}(f) \ggg \operatorname{arr}(g) & =(\operatorname{arr}(g) \mid I) \bullet(I \mid \operatorname{arr}(f)) \\
& \stackrel{(2)}{=} \quad \mu \circ T(\eta \circ(\mathrm{id} \otimes g)) \circ \eta \circ(\mathrm{id} \otimes f) \\
& =T(\mathrm{id} \otimes g) \circ \eta \circ(\mathrm{id} \otimes f) \\
& =\eta \circ(\mathrm{id} \otimes g) \circ(\mathrm{id} \otimes f) \\
& =\eta \circ(\mathrm{id} \otimes(g \circ f)) \\
& \stackrel{(2)}{=} \quad \operatorname{arr}(g \circ f) \mid I \\
& =I \mid \operatorname{arr}(g \circ f) \\
& \stackrel{\lambda}{\cong} \operatorname{arr}(g \circ f) .
\end{aligned}
$$


2 Similarly,

$$
\begin{aligned}
T(\rho \otimes \mathrm{id}) \circ(c \gg \operatorname{arr}(g)) & =T(\rho \otimes \mathrm{id}) \circ((X \mid \operatorname{arr}(g)) \bullet(c \mid I)) \\
& \stackrel{(2)}{=} T(\rho \otimes \mathrm{id}) \circ \mu \circ T(\eta \circ(\mathrm{id} \otimes g)) \circ(c \mid I) \\
& =T(\rho \otimes \mathrm{id}) \circ T(\mathrm{id} \otimes g) \circ(c \mid I) \\
& =T(\mathrm{id} \otimes g) \circ T(\rho \otimes \mathrm{id}) \circ(c \mid I) \\
& \stackrel{(4)}{=} T(\mathrm{id} \otimes g) \circ(c \mid I) \circ(\rho \otimes \mathrm{id}) .
\end{aligned}
$$

3 Associativity of $\gg$ follows from a straightforward calculation that is best done in the Kleisli category:

$$
\begin{aligned}
& (\alpha \otimes \mathrm{id}) \bullet(c \gg(d \gg e)) \\
& =(\alpha \otimes \mathrm{id}) \bullet(X \mid d \gg e) \bullet(c \mid Y \otimes Z) \\
& =(\alpha \otimes \mathrm{id}) \bullet(X \mid((Y \mid e) \bullet(d \mid Z))) \bullet(c \mid Y \otimes Z) \\
& \stackrel{(3)}{=}(\alpha \otimes \mathrm{id}) \bullet(X \mid(Y \mid e)) \bullet(X \mid(d \mid Z)) \bullet(c \mid Y \otimes Z) \\
& \stackrel{(5)}{=}(X \otimes Y \mid e) \bullet(\alpha \otimes \mathrm{id}) \bullet(X \mid(d \mid Z)) \bullet(c \mid Y \otimes Z) \\
& \stackrel{(6)}{=}(X \otimes Y \mid e) \bullet(X \mid d) \mid Z) \bullet(\alpha \otimes \mathrm{id}) \bullet(c \mid Y \otimes Z) \\
& \stackrel{(5)}{=}(X \otimes Y \mid e) \bullet((X \mid d) \mid Z) \bullet((c \mid Y) \mid Z) \bullet(\alpha \otimes \mathrm{id}) \\
& \stackrel{(3)}{=}(X \otimes Y \mid e) \bullet(((X \mid d) \bullet(c \mid Y)) \mid Z) \bullet(\alpha \otimes \mathrm{id}) \\
& =(X \otimes Y \mid e) \bullet(c \gg d \mid Z) \bullet(\alpha \otimes \mathrm{id}) \\
& =((c \gg d) \gg e) \bullet(\alpha \otimes \mathrm{id}) \text {. }
\end{aligned}
$$

4 We only prove functoriality, in a direct way: for two maps of coalgebraic components $f: c_{1} \rightarrow c_{2}$ and $g: d_{1} \rightarrow d_{2}$, where $c_{i}: X_{i} \otimes A \rightarrow T\left(X_{i} \otimes B\right)$ and $d_{i}: Y_{i} \otimes B \rightarrow T\left(Y_{i} \otimes X\right)$ the map $f \otimes g: X_{1} \otimes Y_{1} \rightarrow X_{2} \otimes Y_{2}$ is a morphism of composite coalgebraic components:

$$
\begin{aligned}
& \left((f \otimes g) \otimes \mathrm{id}_{C}\right) \bullet\left(c_{1} \ggg d_{1}\right)=\left((f \otimes g) \otimes \mathrm{id}_{C}\right) \bullet\left(X_{1} \mid d_{1}\right) \bullet\left(c_{1} \mid Y_{1}\right) \\
& \left.\stackrel{(7)}{=}\left(X_{2} \mid d_{2}\right) \bullet\left((f \otimes g) \otimes \operatorname{id}_{B}\right)\right) \bullet\left(c_{1} \mid Y_{1}\right) \\
& \stackrel{(7)}{=}\left(X_{2} \mid d_{2}\right) \bullet\left(c_{2} \mid Y_{2}\right) \bullet\left((f \otimes g) \otimes \operatorname{id}_{A}\right) \\
& =\left(c_{2} \gg d_{2}\right) \bullet\left((f \otimes g) \otimes \operatorname{id}_{A}\right) \text {. }
\end{aligned}
$$

The next result captures the interaction between sequential composition $\gg$ and state extension $\mid$.

Lemma 4.3. For components $X \otimes A \stackrel{c}{\rightarrow} T(X \otimes B)$ to $Y \otimes B \stackrel{d}{\rightarrow} T(Y \otimes C)$ there are associativity isomorphisms:

$$
(U \mid(c \gg d)) \stackrel{\alpha}{\cong}((U \mid c) \gg d) \text { and }(c \gg(d \mid V)) \stackrel{\alpha}{\cong}((c \gg d) \mid V) .
$$


Proof. By the properties of Lemma 3.2. We only do the first one.

$$
\begin{aligned}
& (\alpha \otimes \mathrm{id}) \bullet(U \mid(c \gg d))=(\alpha \otimes \mathrm{id}) \bullet(U \mid((X \mid d) \bullet(c \mid Y))) \\
& \stackrel{(7)}{=}(\alpha \otimes \mathrm{id}) \bullet(U \mid(X \mid d)) \bullet(U \mid(c \mid Y)) \\
& \stackrel{(5)}{=}((U \otimes X) \mid d) \bullet(\alpha \otimes \mathrm{id}) \bullet(U \mid(c \mid Y)) \\
& \stackrel{(6)}{=}((U \otimes X) \mid d) \bullet((U \mid c) \mid Y) \bullet(\alpha \otimes \mathrm{id}) \\
& =((U \mid c) \gg d) \bullet(\alpha \otimes \mathrm{id}) \text {. }
\end{aligned}
$$

\subsection{Multiplicative parallel composition}

Two coalgebraic components $c, d$, with different state spaces, and different inputs \& outputs, can be put in parallel to form new components. This can be done in different ways. At this stage we briefly discuss the "multiplicative" way of doing so, by taking the tensor of the inputs \& outputs. Later on we also describe the "additive" parallel composition that involves the coproduct of inputs \& outputs. This additive version turns out to be more important in the current setting. Our discussion of the multiplicative version will thus be rather brief.

Definition 4.4. For components $X \otimes A \stackrel{c}{\rightarrow} T(X \otimes B)$ and $Y \otimes C \stackrel{d}{\rightarrow} T(Y \otimes D)$ the multiplicative parallel composition $c \| d$ is defined as Kleisli composition:

$$
\begin{gathered}
(X \otimes Y) \otimes(A \otimes C) \stackrel{c \| d}{\longrightarrow}(X \otimes Y) \otimes(B \otimes D) \\
\widehat{\gamma} \downarrow \cong \\
\cong \uparrow \widehat{\gamma} \\
(X \otimes A) \otimes(Y \otimes C) \stackrel{c \otimes d}{\longrightarrow}(X \otimes B) \otimes(Y \otimes D)
\end{gathered}
$$

where $\widehat{\gamma}$ is the obvious isomorphism that swaps the inner two objects.

It is easy to see that $\|$ yields a functor

$$
\| \quad: \quad \operatorname{Comp}(T, A, B) \times \operatorname{Comp}(T, C, D) \longrightarrow \operatorname{Comp}(T, A \otimes C, B \otimes D) .
$$

In the (multi-sorted) Lawvere theory notation from (Hasuo et al., 2009) this operator can be described as a map $\|:(A, B) \times(C, D) \rightarrow(A \otimes C, B \otimes D)$.

Remark 4.5. The type (7) of the functor $\|$ even suggests that the correspondence $\operatorname{Comp}(T,-,-)$ be a (lax) monoidal functor $\mathbb{C}^{\text {op }} \times \mathbb{C} \rightarrow$ Cat, where the former has an obvious monoidal structure (inherited from $\mathbb{C}$ ) and the latter has Cartesian products as tensor products. This is also true of the additive parallel composition functor $\square$ studied later in Section 4.2. The use of such higher-dimensional structures is however not clear at this stage; therefore the relevant technical developments are left as future work.

Now that we have this $\|$ operator we can describe the equivalents of the 'first' and 'second' operators in the context of Hughes' Arrows (Hughes, 2000). They add an additional input \& output, on the left or on the right of the existing input \& output, namely 
via

$$
\operatorname{first}_{\|}(c)=c \| \operatorname{arr}(\mathrm{id}) \quad \text { and } \quad \operatorname{second}_{\|}(c)=\operatorname{arr}(\mathrm{id}) \| c .
$$

We only mention the following result about $\|$, without proof.

Lemma 4.6. There are isomorphisms of components:

$$
(U \mid(c \| d)) \stackrel{\alpha}{\cong}((U \mid c) \| d) \text { and } \quad(c \|(d \mid V)) \stackrel{\alpha}{\cong}((c \| d) \mid V) .
$$

\subsection{Additive parallel composition}

The next aim is to define an additive parallel composition operator $\square$ for coalgebraic components. It is called 'external choice' in (Barbosa, 2001; Barbosa, 2003). We need to assume that our category $\mathbb{C}$ has binary coproducts + , and that the tensor $\otimes$ distributes over them, as described in Section 2, via a distribution map dis as in (2). These coproducts + in $\mathbb{C}$ also form coproducts in the Kleisli category $\mathcal{K} \ell(T)$ and are preserved by $J: \mathbb{C} \rightarrow$ $\mathcal{K} \ell(T)$. Thus in $\mathcal{K} \ell(T)$ one has coprojections $J\left(\kappa_{i}\right)=\eta \circ \kappa_{i}: X_{i} \rightarrow T\left(X_{1}+X_{2}\right)$ with cotupling as in $\mathbb{C}$. Since the monad $T$ is assumed to be commutative (i.e. symmetric monoidal $), \otimes$ is also a tensor in $\mathcal{K} \ell(T)$, and it distributes over + in $\mathcal{K} \ell(T)$, via $J($ dis $)=$ $\eta \circ$ dis as distributivity isomorphism.

Definition 4.7. An additive parallel operator $\square$ is defined on coalgebraic components $X \otimes A \stackrel{c}{\rightarrow} T(X \otimes B)$ and $Y \otimes C \stackrel{d}{\rightarrow} T(Y \otimes D)$ as Kleisli composition:

$$
\begin{aligned}
& (X \otimes Y) \otimes(A+C) \longrightarrow(X \otimes Y) \otimes(B+D) \\
& \left.\operatorname{dis}^{-1}\right\rfloor \cong \quad \cong \uparrow \text { dis } \\
& (X \otimes Y) \otimes A+(X \otimes Y) \otimes C \stackrel{c|Y+X| d}{\longrightarrow}(X \otimes Y) \otimes B+(X \otimes Y) \otimes D
\end{aligned}
$$

where $c|Y+X| d$ is the coproduct of maps in the Kleisli category.

This additive composition operator $\square$ forms a functor $\operatorname{Comp}(T, A, B) \times \operatorname{Comp}(T, C, D) \rightarrow$ $\operatorname{Comp}(T, A+C, B+D)$, which is defined on morphisms as $f \square g=f \otimes g$. It may thus be written as a map $\square:(A, B) \times(C, D) \rightarrow(A+C, B+D)$. This will be helpful in Section 6 .

Just like we had 'first' and 'second' operator for multiplicative parallel composition (8), we also have them in the additive case:

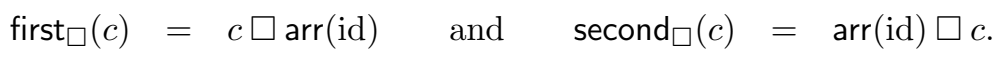

These fundamental operators occur frequently in the sequel, for instance in the (di)naturality properties of the trace operator in Section 6 .

We make the relation between $\square$ and state extension explicit. It is very similar to the relations between $\gg$ or $\|$ and state extension, see Lemma 4.3 and Lemma 4.6.

Lemma 4.8. For components $X \otimes A \stackrel{c}{\rightarrow} T(X \otimes B)$ to $Y \otimes C \stackrel{d}{\rightarrow} T(Y \otimes D)$ there are associativity isomorphisms:

$$
(U \mid(c \square d)) \stackrel{\alpha}{\cong}((U \mid c) \square d) \quad \text { and } \quad(c \square(d \mid V)) \stackrel{\alpha}{\cong}((c \square d) \mid V) .
$$


Proof. This requires a rather elaborate calculation, which for the first one looks as follows.

$$
\begin{aligned}
& ((U \mid c) \square d) \bullet(\alpha \otimes \mathrm{id}) \\
& =\quad \operatorname{dis} \bullet(((U \mid c) \mid Y)+((U \otimes X) \mid d)) \bullet \operatorname{dis}^{-1} \bullet(\alpha \otimes \mathrm{id}) \\
& \stackrel{2.1 .1}{=} \operatorname{dis} \bullet(((U \mid c) \mid Y)+((U \otimes X) \mid d)) \bullet((\alpha \otimes \mathrm{id})+(\alpha \otimes \mathrm{id})) \bullet \mathrm{dis}^{-1} \\
& \stackrel{2.1 .2}{=} \operatorname{dis} \bullet((((U \mid c) \mid Y) \bullet(\alpha \otimes \mathrm{id}))+(((U \otimes X) \mid d) \bullet(\alpha \otimes \mathrm{id}))) \bullet \\
& (\alpha+\alpha) \bullet \operatorname{dis}^{-1} \bullet\left(U \otimes \operatorname{dis}^{-1}\right) \bullet \alpha^{-1} \\
& \stackrel{3.2 .6,(5)}{=} \operatorname{dis} \bullet(((\alpha \otimes \mathrm{id}) \bullet(U \mid(c \mid Y)) \bullet \alpha)+((\alpha \otimes \mathrm{id}) \bullet(U \mid(X \mid d)) \bullet \alpha)) \bullet \\
& \operatorname{dis}^{-1} \bullet\left(U \otimes \operatorname{dis}^{-1}\right) \bullet \alpha^{-1} \\
& =\operatorname{dis} \bullet((\alpha \otimes \mathrm{id})+(\alpha \otimes \mathrm{id})) \bullet \\
& \left(\left(\alpha \bullet(U \otimes(c \mid Y)) \bullet \alpha^{-1} \bullet \alpha\right)+\left(\alpha \bullet(U \otimes(X \mid d)) \bullet \alpha^{-1} \bullet \alpha\right)\right) \bullet \\
& \operatorname{dis}^{-1} \bullet\left(U \otimes \operatorname{dis}^{-1}\right) \bullet \alpha^{-1} \\
& \stackrel{2.1 .1}{=}(\alpha \otimes \mathrm{id}) \bullet \operatorname{dis} \bullet(\alpha+\alpha) \bullet((U \otimes(c \mid Y))+(U \otimes(X \mid d))) \bullet \\
& \operatorname{dis}^{-1} \bullet\left(U \otimes \operatorname{dis}^{-1}\right) \bullet \alpha^{-1} \\
& \stackrel{2.1 .2}{=}(\alpha \otimes \mathrm{id}) \bullet \alpha \bullet(U \otimes \mathrm{dis}) \bullet \operatorname{dis} \bullet((U \otimes(c \mid Y))+(U \otimes(X \mid d))) \bullet \\
& \operatorname{dis}^{-1} \bullet\left(U \otimes \operatorname{dis}^{-1}\right) \bullet \alpha^{-1} \\
& \stackrel{2.1 .1}{=}(\alpha \otimes \mathrm{id}) \bullet \alpha \bullet(U \otimes \mathrm{dis}) \bullet(U \otimes(c|Y+X| d)) \bullet\left(U \otimes \mathrm{dis}^{-1}\right) \bullet \alpha^{-1} \\
& =(\alpha \otimes \mathrm{id}) \bullet \alpha \bullet(U \otimes(c \square d)) \bullet \alpha^{-1} \\
& =(\alpha \otimes \mathrm{id}) \bullet(U \mid(c \square d)) \text {. }
\end{aligned}
$$

Many more properties, like associativity, can be proven about $\square$. We explicitly state some of them, leaving the details of the (straightforward) proof to the reader.

\section{Lemma 4.9 .}

1 Given arrows $f: A \rightarrow B$ and $g: C \rightarrow D$ in $\mathbb{C}$, we have a canonical isomorphism of coalgebraic components $\operatorname{arr}(f) \square \operatorname{arr}(g) \stackrel{\cong}{\longrightarrow} \operatorname{arr}(f+g)$. That is,

$$
\begin{aligned}
& T((I \otimes I) \otimes(B+D)) \stackrel{T(\lambda \otimes \mathrm{id})=T(\rho \otimes \mathrm{id})}{\cong} T(I \otimes(B+D)) \\
& \begin{array}{r}
\text { arr }(f) \square \operatorname{arr}(g) \uparrow \\
(I \otimes I) \otimes(A+C) \frac{\operatorname{arr}(f+g)}{\lambda \otimes \mathrm{id}=\rho \otimes \mathrm{id}} \\
\cong
\end{array} \quad I \otimes(A+C)
\end{aligned}
$$


2 One has $\left(c \gg c^{\prime}\right) \square\left(d \gg d^{\prime}\right) \stackrel{\cong}{\longrightarrow}(c \square d) \ggg\left(c^{\prime} \square d^{\prime}\right)$, in:

$$
\begin{aligned}
& T\left(\left(X \otimes X^{\prime}\right) \otimes\left(Y \otimes Y^{\prime}\right) \otimes\left(A^{\prime \prime}+B^{\prime \prime}\right)\right)_{T(\beta \otimes \mathrm{id})} \\
& \left(c \gg c^{\prime}\right) \square\left(d \gg d^{\prime}\right) \uparrow \stackrel{T(\beta \otimes \mathrm{id})}{\cong} T\left((X \otimes Y) \otimes\left(X_{\uparrow}^{\prime} \otimes Y^{\prime}\right) \otimes\left(A^{\prime \prime}+B^{\prime \prime}\right)\right) \\
& \left(X \otimes X^{\prime}\right) \otimes\left(Y \otimes Y^{\prime}\right) \otimes(A+B) \quad \beta \otimes \mathrm{id} \quad(c \square d) \ggg\left(c^{\prime} \square d^{\prime}\right) \\
& (X \otimes Y) \otimes\left(X^{\prime} \otimes Y^{\prime}\right) \otimes(A+B)
\end{aligned}
$$

Here $\beta:\left(X \otimes X^{\prime}\right) \otimes\left(Y \otimes Y^{\prime}\right) \stackrel{\cong}{\longrightarrow}(X \otimes Y) \otimes\left(X^{\prime} \otimes Y^{\prime}\right)$ is the canonical isomorphism in a symmetric monoidal category $(\mathbb{C}, \otimes, I)$.

3 One has $(c \square(d \square e)) \gg \operatorname{arr}\left(\alpha_{+}\right) \stackrel{\cong}{\longrightarrow} \operatorname{arr}\left(\alpha_{+}\right) \gg((c \square d) \square e)$, in:

$$
\begin{gathered}
T\left(( ( X \otimes ( Y \otimes Z ) ) \otimes I ) \otimes \left(\left(D+\frac{E)+F))_{T\left(\beta^{\prime} \otimes \mathrm{id}\right)}}{\cong} T((I \otimes((X \otimes Y) \otimes Z)) \otimes((D+E)+F))\right.\right.\right. \\
(c \square(d \square e)) \ggg \operatorname{arr}\left(\alpha_{+}\right) \mid \\
((X \otimes(Y \otimes Z)) \otimes I) \otimes\left(A+\frac{(B+C))_{\beta^{\prime} \otimes \mathrm{id}}}{\cong}(I \otimes(X \otimes Y) \otimes Z)\right) \otimes(A+(B+C))
\end{gathered}
$$

Here $\beta^{\prime}:(X \otimes(Y \otimes Z)) \otimes I \cong I \otimes((X \otimes Y) \otimes Z)$ is the canonical isomorphism in $\mathbb{C}$; $\alpha_{+}$is the associativity isomorphism for + .

4 One has $(c \square d) \gg \operatorname{arr}\left(\gamma_{+}\right) \stackrel{\cong}{\longrightarrow} \operatorname{arr}\left(\gamma_{+}\right) \gg(d \square c)$, in:

$$
\begin{aligned}
& T(((X \otimes Y) \otimes I) \otimes(D+C)) \stackrel{T\left(\beta^{\prime \prime} \otimes \mathrm{id}\right)}{\cong} T((I \otimes(Y \otimes X)) \otimes(D+C)) \\
& (c \square d) \ggg \operatorname{arr}\left(\gamma_{+}\right) \uparrow \quad \uparrow \operatorname{arr}\left(\gamma_{+}\right) \ggg(d \square c) \\
& ((X \otimes Y) \otimes I) \otimes(A+B) \underset{\cong}{\stackrel{\beta^{\prime \prime} \otimes \mathrm{id}}{\cong}}(I \otimes(Y \otimes X)) \otimes(A+B)
\end{aligned}
$$

Here $\beta^{\prime \prime}=\lambda^{-1} \circ \gamma \circ \rho:(X \otimes Y) \otimes I \stackrel{\cong}{\longrightarrow} I \otimes(Y \otimes X)$ and $\gamma_{+}$is the symmetry isomorphism for + .

Proof. Items $1-2$ are easy by direct calculation. Items $3-4$ follow essentially from naturality of $\alpha_{+}$and $\gamma_{+}$, and Lemma 2.2.2-3.

\subsection{Method combination}

The additive parallel composition $c \square d$ from the previous subsection applies to arbitrary components $c, d$, which typically have difference state spaces. In the special case when $c, d$ share the same state space, there is also a composition operator which we shall write as $\{c, d\}$. It can be seen as a combination of the (Java-like) methods of $c$ and $d$ on their shared state space. 
Definition 4.10. For two components $X \otimes A \stackrel{c}{\rightarrow} T(X \otimes B)$ to $X \otimes C \stackrel{d}{\rightarrow} T(X \otimes D)$ with the same state space $X$ we define $\{c, d\}$ as Kleisli composition:

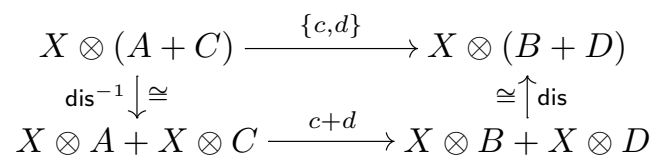

If we understand a coalgebraic component as a mathematical model of a class in an object oriented programming language, then we can see this method combination operator as a form of building classes: first the state space $X$ is fixed, and subsequently methods $c_{i}: X \otimes A_{i} \rightarrow T\left(X \otimes B_{i}\right)$ are combined to a class $c=\left\{c_{1}, \ldots, c_{n}\right\}: X \otimes\left(A_{1}+\right.$ $\left.\cdots+A_{n}\right) \rightarrow T\left(X \otimes\left(B_{1}+\cdots+B_{n}\right)\right)$.

Moreover, extension of classes can be described, yielding a form of subclass and inheritance, albeit without overriding of methods. Given a class/component $c: X \otimes A \rightarrow$ $T(X \otimes B)$ one can form a subclass by first extending the state to $c \mid Y:(X \otimes Y) \otimes A \rightarrow$ $T((X \otimes Y) \otimes B)$. Indeed, subclassing involves an extended state, to accommodate for additional fields/attributes. Additional methods $d:(X \otimes Y) \otimes C \rightarrow T((X \otimes Y) \otimes D)$ may now be added to obtain a subclass $c^{\prime}=\{c \mid Y, d\}:(X \otimes Y) \otimes(A+C) \rightarrow T((X \otimes Y) \otimes(B+D))$.

The proof of the following result is left to the reader.

Lemma 4.11. Method combination commutes with state extension:

$$
U \mid\{c, d\}=\{U|c, U| d\} \quad \text { and } \quad\{c, d\} \mid V=\{c|V, d| V\} .
$$

\section{Tube diagrams for components}

Our goal in the next section (which is central in this paper) is: to first introduce a trace operator that realises feedback loops for components; and then to prove that the introduced operator indeed satisfies the expected equational properties from (Joyal et al., 1996) - such as dinaturality, yanking and superposing. It turns out, however, that the composed components occurring in the equations are rather complicated and their structures at large are best depicted using a certain variant of string diagrams.

String diagrams were introduced in (Penrose, 1971) for succinctly depicting morphisms in a monoidal category; see also (Joyal and Street, 1991). The trouble here is that we need to deal with two different kinds of monoidal structure $\otimes$ and + ; this calls for a carefully devised pictorial convention. Following (McCurdy, 2010), we employ the version of string diagrams that are augmented by tubes - we refer to them as tube diagrams. Tubes enhance a slightly more common pictorial convention of functorial boxes (Cockett and Seely, 1999; Melliès, 2006). In the current paper, tubes capture applications of functors of the form $X \otimes-$.

We now present tube diagrams (based on (McCurdy, 2010)) for some of the composition operators introduced previously in Section 4. We start with drawing some diagrams; explanations follow shortly. First consider the sequential composition operator in Definition 4.1. Given two components

$$
X \otimes A \stackrel{c}{\longrightarrow} X \otimes B \quad \text { and } \quad Y \otimes B \stackrel{d}{\longrightarrow} Y \otimes C \quad \text { in } \mathcal{K} \ell(T)
$$


with matching input and output, each of these shall be depicted as follows.

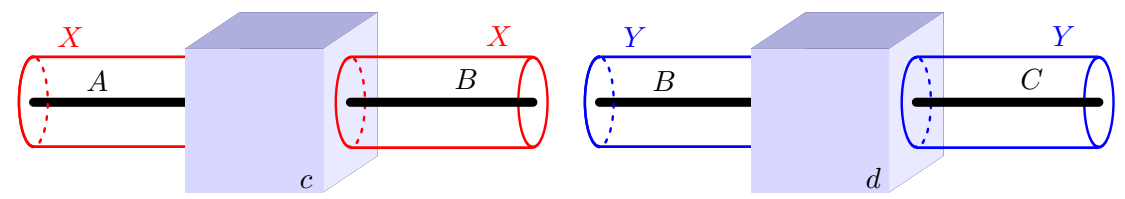

Their composition $(X \otimes Y) \otimes A \stackrel{c \ggg d}{\longrightarrow}(X \otimes Y) \otimes C$ is then depicted as follows.

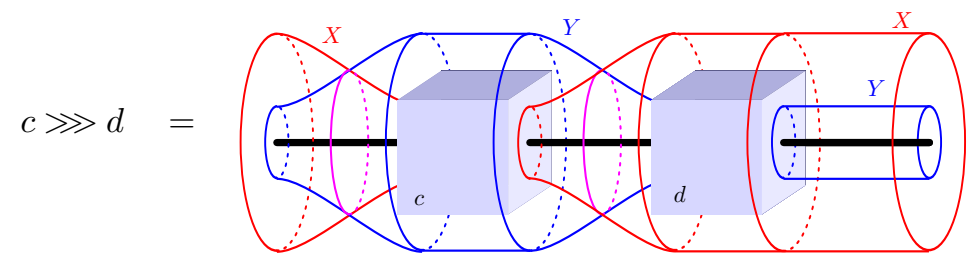

The conventions are as follows.

- Diagrams are read from left to right.

— Each tube designates an object in $\mathcal{K} \ell(T)$; more precisely it designates the identity morphism on the object. In the last diagram (10), the (red) tube that is shrunk and plugged into the $c$-box, as well as the one that comes out of $c$ and is expanded to enclose the $d$-box, are of type $X$. Other two (blue) tubes - the one that encloses $c$ and the one that comes out of $d$-are of type $Y$. The three thick lines (in black) are of type $A, B$ or $C$. These "lines" are tubes in fact; they are depicted as lines solely for the purpose of simplicity of the picture.

- Nested tubes designate the tensor $\otimes$ in $\mathcal{K} \ell(T)$, calculated from the outermost one towards inside. For example, the left endpoint of the last diagram (a red tube, a blue one and then a black one, going inwards) designates the object $X \otimes Y \otimes A$.

- Symmetry $\gamma$ of the tensor $\otimes$ is depicted as a "waist", i.e. the exchange of the outer tube and the inner tube. This occurs twice in the last diagram where the "waists" are marked with (pink) circles.

- Associativity isomorphisms are left implicit. That is, when dealing with tube diagrams we assume strict monoidal structures where we do not distinguish $(X \otimes Y) \otimes A$ from $X \otimes(Y \otimes A)$, nor $(X+Y)+A$ from $X+(Y+A)$. For this reason our later use of tube diagrams is as "guidelines" for rigorous calculational proofs, rather than as proofs by themselves. We will come back to this point later in Remark 5.2.

To summarise, the last tube diagram (10) is "parsed" into the following composition of morphisms in $\mathcal{K} \ell(T)$.

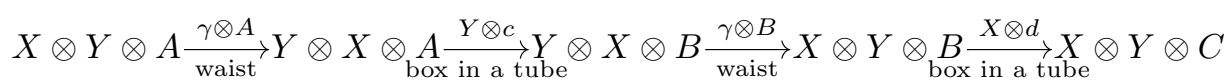

This composition is therefore the same thing as in Definition 4.1, modulo the use of associativity isomorphisms $\alpha$. It is implicit in this correspondence that left- and right- 
state extensions (Definition 3.1) can be depicted as follows.
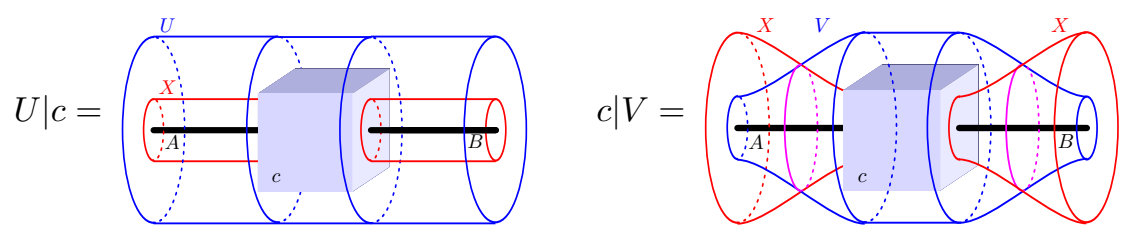

The "waist" diagram that represents symmetry $\gamma: X \otimes Y \cong Y \otimes X$ might seem strange at the first sight. In fact, one recovers the usual "crossing" representation of symmetry (see e.g. (Joyal and Street, 1991)) by looking at a certain section of the three-dimensional picture $^{\dagger}$, as in:

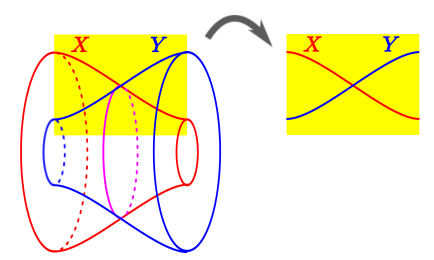

As opposed to the multiplicative tensor $\otimes$, the additive tensor + is depicted by putting two tubes in parallel (rather than nested). That is for example:

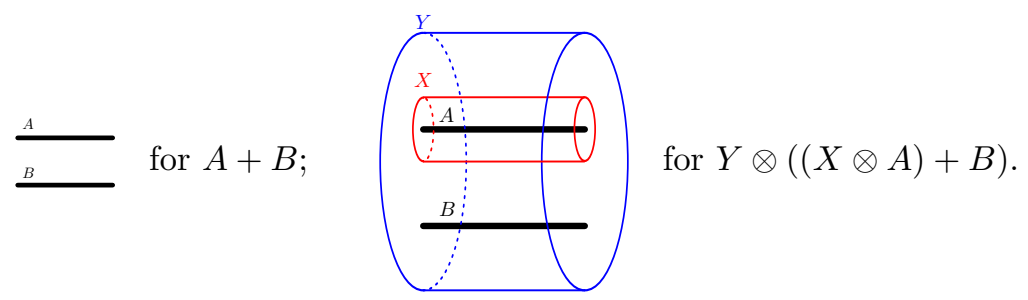

Relating the two tensors $\otimes$ and + is the distributivity isomorphism dis from (2). It has a nice graphical representation as a pair of "pants":

$$
\text { dis : } X \otimes A+X \otimes B \longrightarrow X \otimes(A+B) \text { as }
$$

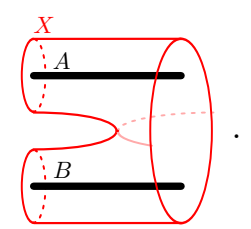

Furthermore, dis being an isomorphism means that the following equalities hold. The right hand sides are identity maps on suitable objects.
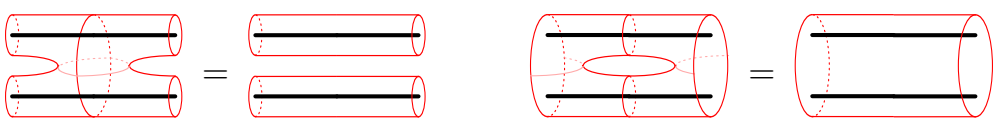

We shall establish another couple of lemmas for manipulating such distributivity "pants".

$\dagger$ This observation is due to Shin-ya Katsumata. 
Lemma 5.1 (Mr. Bean’s Pants Exchange ${ }^{\ddagger}$ ). The following diagram commutes:

$$
\begin{aligned}
& X \otimes(Y \otimes A)+X \otimes(Y \otimes B) \stackrel{\text { dis }}{\longrightarrow} X \otimes(Y \otimes A+Y \otimes B) \stackrel{X \otimes \text { dis }}{\longrightarrow} X \otimes(Y \otimes(A+B)) \\
& \alpha+\alpha \downarrow \\
& (X \otimes Y) \otimes A+(X \otimes Y) \otimes B \\
& \gamma \otimes A+\gamma \otimes B \downarrow \\
& (Y \otimes X) \otimes A+(Y \otimes X) \otimes B \\
& \alpha^{-1}+\alpha^{-1} \downarrow \\
& \downarrow \alpha \\
& (X \otimes Y) \otimes(A+B) \\
& \downarrow \gamma \otimes(A+B) \\
& (Y \otimes X) \otimes(A+B) \\
& \downarrow \alpha^{-1} \\
& Y \otimes(X \otimes A)+Y \otimes(X \otimes B) \stackrel{\text { dis }}{\longrightarrow} Y \otimes(X \otimes A+X \otimes B) \stackrel{Y \otimes \text { dis }}{\longrightarrow} Y \otimes(X \otimes(A+B))
\end{aligned}
$$

which, in a strict monoidal category, is reduced to the following one:

$$
\begin{aligned}
& X \otimes Y \otimes A+X \otimes Y \otimes B \stackrel{\text { dis }}{\longrightarrow} X \otimes(Y \otimes A+Y \otimes B) \stackrel{\text { X®dis }}{\longrightarrow} X \otimes Y \otimes(A+B) \\
& \quad \gamma \otimes A+\gamma \otimes B \downarrow \\
& Y \otimes X \otimes A+Y \otimes X \otimes B \stackrel{\text { dis }}{\longrightarrow} Y \otimes(X \otimes A+X \otimes B) \stackrel{Y \otimes \text { dis }}{\longrightarrow} Y \otimes X \otimes(A+B)
\end{aligned}
$$

Recall that $\gamma$ denotes symmetry isomorphisms for $\otimes$. This is in terms of tube diagrams:

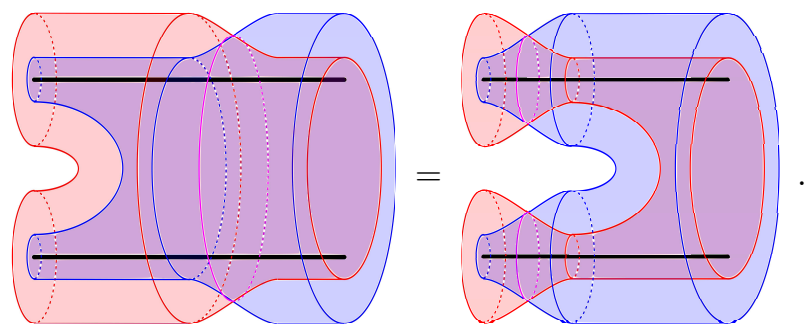

Proof. By drawing two horizontal maps labelled with dis in the above diagram in the lemma, using Lemma 2.2.3 twice, and naturality for dis from Lemma 2.1.1.

Next we note that the interaction between distribution dis and the coproduct associativity $\alpha_{+}$from Lemma 2.2 .3 is equivalent to the following one, in a strict monoidal setting.

$$
\begin{gathered}
X \otimes A+X \otimes(B+C) \underset{\text { dis }}{\longrightarrow} X \otimes(A+B+C) \\
X \otimes A+\text { dis }^{-1} \downarrow \\
X \otimes A+X \otimes B+X \otimes C \underset{\text { dis }+X \otimes C}{\stackrel{\downarrow}{\longrightarrow}} X \otimes(A+B)+X \otimes C
\end{gathered}
$$

The latter is in terms of tube diagrams:

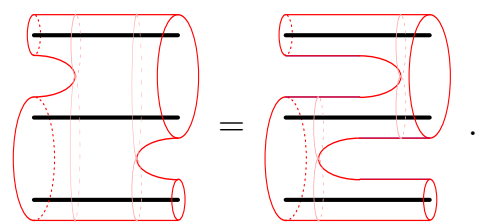

This equality of tubes can be found in (McCurdy, 2010).

$\ddagger$ Mr. Bean, Episode 1, Act 2. 1990. 
Another operator which will be heavily used in Section 6 is the additive parallel composition $\square$. Following Definition 4.7, one composes the diagrams in (11) and (12) to obtain the following tube diagram for $c \square d$.

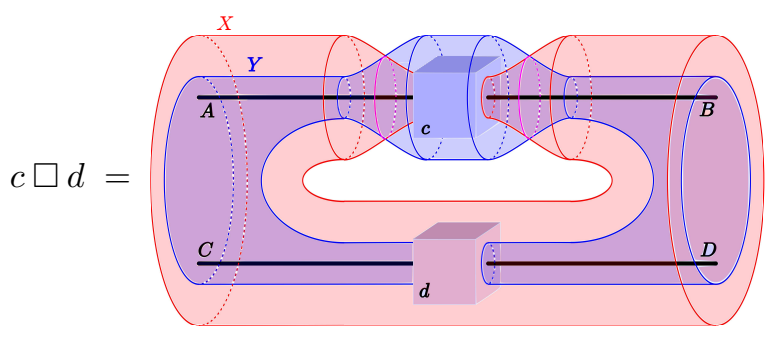

Remark 5.2. To turn our tube-diagram reasoning into mathematically rigorous proofs, one needs a coherence result of one form or another. It can be a statement that any non-strict such category is equivalent to a strict one; or a statement that the category of string/tube diagrams is the free such category. Currently we do not have any of these. This is not a total anomaly: among dozens of well-known graphical languages for various kinds of (monoidal) categories collected in (Selinger, 2010), some are lacking coherence results. Still they offer useful guidelines for rigorous calculational proofs, much like the tube diagrams do in this paper.

Remark 5.3. We emphasise that all tube diagrams represent morphisms in the Kleisli category $\mathcal{K} \ell(T)$. We shall be also employing a different kind of string diagrams later, mostly for describing the trace axioms. The latter kind of diagrams are two-dimensional and are essentially "pictorial shorthands" for composition of components. For example, sequential composition $c \gg d$ of components is represented by

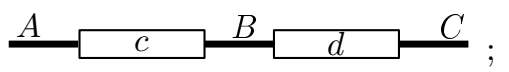

additive parallel composition $c \square d$ is represented by

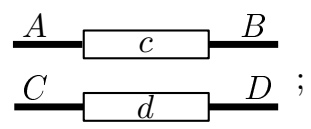

and the "identity component" arr(id) is represented simply by a wire/line. Hence the diagram

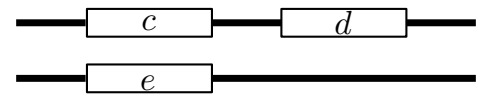

represents the composition $(c \square e) \gg(d \square \operatorname{arr}(\mathrm{id}))$, which is equal to $(c \gg d) \square e$ up-to a canonical isomorphism (this follows from the results in (Asada and Hasuo, 2010; Hasuo et al., 2009)).

In the latter kind of string diagrams wires represent input/output interfaces; state spaces of components are not explicit. To distinguish the two kinds of string diagrams should be easy. In particular, in tube diagrams a component is represented by a 3D shadowed cube, while in the latter a component is a $2 \mathrm{D}$ box. 


\section{A monoidal trace for coalgebraic components}

In (Jacobs, 2010) it is shown how for certain monads $T$ the Kleisli category $\mathcal{K} \ell(T)$ is traced monoidal wrt. coproducts + as monoidal structure. Concretely, this means that for maps of the form $f: X+U \rightarrow T(Y+U)$ there is a trace map $\operatorname{Tr}^{\mathcal{K} \ell}(f): X \rightarrow T(Y)$ satisfying standard properties, see (Joyal et al., 1996). This trace operator $\operatorname{Tr}^{\mathcal{K} \ell}$ on the Kleisli category will be used to construct a similar trace operator for coalgebraic components. The main task in this section is to show that the trace properties from (Joyal et al., 1996) also hold for these components, but only up to (canonical) isomorphism.

The precise properties that $T$ must satisfy to obtain this (Kleisli) trace operator $\operatorname{Tr}^{K \ell}$ are listed in (Jacobs, 2010, Requirements 4.7). The main ones are that the category $\mathbb{C}$ should have (countable) coproducts, the Kleisli category should be enriched over the category of dcpo's with bottom, and the monad should be 'semi-additive'. In this section we shall simply assume that these properties hold for $T$. Examples of a such monad $T$ include: the lift monad $T=1+(-)$ for partiality; the powerset monad $\mathcal{P}$ for nondeterminism as well as its bounded variant $\mathcal{P}<\kappa$ with $\kappa>\aleph_{0}$; and the (discrete, countable) subdistribution monad $\mathcal{D}$ for probabilistic non-determinism where

$$
\mathcal{D} X=\left\{d: X \rightarrow[0,1] \mid \sum_{x \in X} d(x) \leq 1\right\}
$$

Such a $d$ is a "sub" distribution since its sum is $\leq 1$, rather than $=1$ (see e.g. (Hasuo et al., 2007)).

Definition 6.1. The trace operator $\operatorname{Tr}:(A+C, B+C) \rightarrow(A, B)$ is defined on a coalgebraic component $c: X \otimes(A+C) \rightarrow T(X \otimes(B+C))$ via the Kleisli trace operator $\operatorname{Tr}^{K \ell}$

$$
\operatorname{Tr}(c) \stackrel{\text { def }}{=} \operatorname{Tr} \mathcal{K}\left(X \otimes A+X \otimes C \stackrel{T\left(\mathrm{dis}^{-1}\right) \circ c \circ \text { dis }}{\longrightarrow} T(X \otimes B+X \otimes C)\right) .
$$

Notice that this composition inside $\operatorname{Tr}^{\mathcal{K} \ell}(-)$ is really a Kleisli composition.

Using the tube diagram scheme that we described in Section 5, the trace operator is depicted in the following way. First, the composite inside the trace operator $\operatorname{Tr}^{K \ell}$ is

$$
\operatorname{dis}^{-1} \bullet c \bullet \operatorname{dis} \quad: \quad X \otimes A+X \otimes B \longrightarrow X \otimes B+X \otimes C \quad \text { in } \mathcal{K} \ell(T),
$$

hence is depicted as follows.

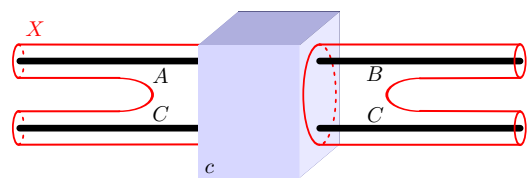

Applying the trace operator $\operatorname{Tr}^{\mathcal{K} \ell}$ yields

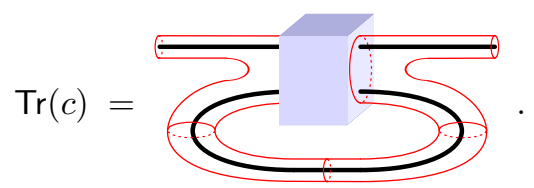

Functoriality of the operator $\operatorname{Tr}$ is essential. 
Lemma 6.2. The trace operator Tr extends to a functor

$$
\operatorname{Tr} \quad: \operatorname{Comp}(T, A+C, B+C) \longrightarrow \operatorname{Comp}(T, A, B) .
$$

That is, given two components

$$
c: X \otimes(A+C) \rightarrow T(X \otimes(B+C)), \quad d: Y \otimes(A+C) \rightarrow T(Y \otimes(B+C))
$$

and a morphism $f$ from $c$ to $d$ (see (4)), $f$ is again a component morphism

$$
\text { from } \operatorname{Tr}(c): X \otimes A \rightarrow T(X \otimes B) \quad \text { to } \operatorname{Tr}(d): Y \otimes A \rightarrow T(Y \otimes B) .
$$

Proof. The result makes essential use of uniformity of the trace operator $\operatorname{Tr}^{\mathcal{K} \ell}$ :

$$
(\mathrm{id}+h) \bullet f=g \bullet(\mathrm{id}+h) \text { implies } \operatorname{Tr}^{\mathcal{K} \ell}(f)=\operatorname{Tr}^{\mathcal{K} \ell}(g),
$$

that is, pictorially,

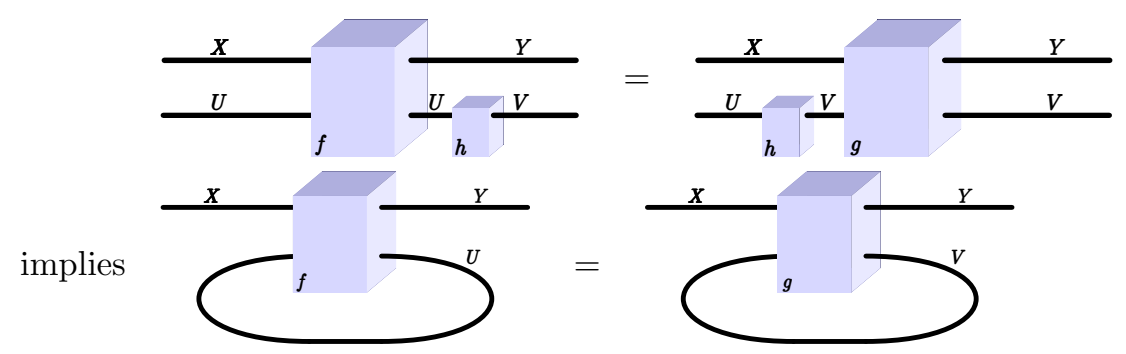

This notion of uniformity for traced monoidal categories is formulated in (Hasegawa, 1999); the name is due to its correspondence to Plotkin's uniformity principle in domain theory (see e.g. (Simpson and Plotkin, 2000)). See (Hasegawa, 2004) for more recent developments as well as more about the historical background.

It is typical that in a traced monoidal category $\mathbb{C}$, uniformity like (19) does not hold for every $h$ but holds only for "strict" $h$. However, when the trace structure of $\mathbb{C}$ arises from $\mathbb{C}$ 's structure as a partially additive category, uniformity is true for every $h$ (Haghverdi, 2000). This is our current setting; see (Jacobs, 2010).

We turn to the proof of the lemma. The following diagram in $\mathcal{K} \ell(T)$ commutes: it is the assumption that $f$ is a component morphism, combined with naturality of dis.

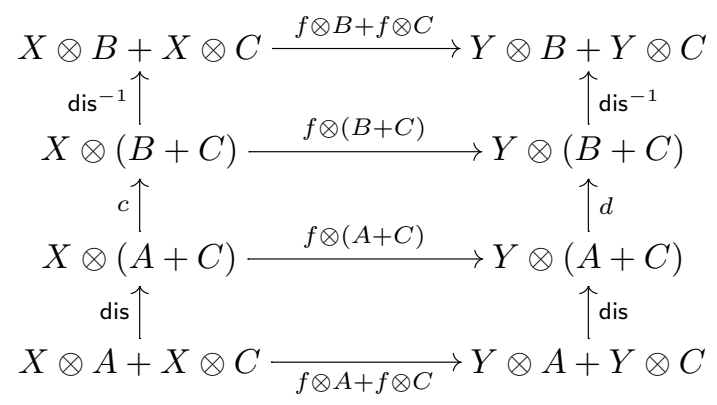

Thus we have

$(\mathrm{id}+f \otimes C) \bullet\left((f \otimes B+\mathrm{id}) \bullet \mathrm{dis}^{-1} \bullet c \bullet \mathrm{dis}\right)=\left(\mathrm{dis}^{-1} \bullet d \bullet \operatorname{dis} \bullet(f \otimes A+\mathrm{id})\right) \bullet(\mathrm{id}+f \otimes C)$, 
from which we derive, by uniformity (19),

$$
\operatorname{Tr}^{\mathcal{K}}\left((f \otimes B+\mathrm{id}) \bullet \operatorname{dis}^{-1} \bullet c \bullet \operatorname{dis}\right)=\operatorname{Tr}^{\mathcal{K}}\left(\operatorname{dis}^{-1} \bullet d \bullet \operatorname{dis} \bullet(f \otimes A+\mathrm{id})\right) .
$$

This is used in the following calculation. It concludes the proof.

$$
\begin{aligned}
& (f \otimes B) \bullet \operatorname{Tr}(c) \\
& =(f \otimes B) \bullet \operatorname{Tr}^{K \ell}\left(\mathrm{dis}^{-1} \bullet c \bullet \mathrm{dis}\right) \\
& =\operatorname{Tr} \mathcal{K l}\left((f \otimes B+\mathrm{id}) \bullet \mathrm{dis}^{-1} \bullet c \bullet \mathrm{dis}\right)
\end{aligned}
$$

by the tightening axiom for the trace operator $\operatorname{Tr}^{K \ell}$, see $e . g$. (Joyal et al., 1996)

$$
\begin{aligned}
& =\operatorname{Tr} \mathcal{K}\left(\operatorname{dis}^{-1} \bullet d \bullet \operatorname{dis} \bullet(f \otimes A+\mathrm{id})\right) \quad \text { by }(20) \\
& =\operatorname{Tr} \mathcal{K}\left(\operatorname{dis}^{-1} \bullet d \bullet \operatorname{dis}\right) \bullet(f \otimes A) \quad \text { by tightening } \\
& =\operatorname{Tr}(d) \bullet(f \otimes A) .
\end{aligned}
$$

We make the following special case explicit.

Lemma 6.3. For an isomorphism $\varphi$ in $\mathbb{C}$ of the appropriate type one has:

$$
(\varphi \otimes \mathrm{id}) \bullet \operatorname{Tr}(c) \bullet\left(\varphi^{-1} \otimes \mathrm{id}\right)=\operatorname{Tr}\left((\varphi \otimes \mathrm{id}) \bullet c \bullet\left(\varphi^{-1} \otimes \mathrm{id}\right)\right) .
$$

Equivalently: if $\varphi$ is an isomorphism of coalgebraic components as on the left (below) then it is also an isomorphism on the right between the corresponding traces:

$$
\begin{aligned}
& T(X \otimes(A+C)) \stackrel{T(\varphi \otimes \mathrm{id})}{\cong} T(Y \otimes(A+C)) \quad T(X \otimes A) \stackrel{T(\varphi \otimes \mathrm{id})}{\cong} T(Y \otimes A)
\end{aligned}
$$

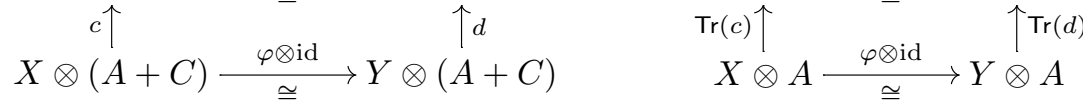

In the remainder of this section we first establish how the component trace Tr interacts with state extension | and with additive parallel composition $\square$, before proving the standard trace properties of (Joyal et al., 1996). The trace properties as well as the preceding lemmas are often accompanied by the intended equalities of tube diagrams. Such tube diagrams hopefully convey some intuition behind rather complicated calculations.

\subsection{Trace and state extension}

In Section 4.2 we have assumed that functors $X \otimes-$ preserve binary coproducts, for instance because $X \otimes-$ has a right adjoint (given by exponents $\multimap$ ).

Proposition 6.4. Let $T$ be a (commutative) monad on a symmetric monoidal category $\mathbb{C}$ with (countable) coproducts, for which the Kleisli category $\mathcal{K} \ell(T)$ has a monoidal trace operator $\operatorname{Tr}^{\mathcal{K}}$ wrt. coproducts. If functors $U \otimes-: \mathbb{C} \rightarrow \mathbb{C}$ preserve coproducts, then $U \otimes-: \mathcal{K} \ell(T) \rightarrow \mathcal{K} \ell(T)$ preserves the trace operator, in the sense that:

$$
U \otimes \operatorname{Tr}^{\mathcal{K} \ell}(X+C \stackrel{f}{\longrightarrow} T(Y+C)): U \otimes X \longrightarrow T(U \otimes Y)
$$


is the same as:

$$
\operatorname{Tr}^{K} \mathcal{L}\left(U \otimes X+U \otimes C \stackrel{\text { dis }}{\cong} U \otimes(X+C) \stackrel{U \otimes f}{\longrightarrow} T(U \otimes(Y+C))^{T\left(\mathrm{dis}^{-1}\right.} \longrightarrow T(U \otimes Y+U \otimes C)\right) .
$$

In terms of tube diagrams, what is asserted is the following equality.

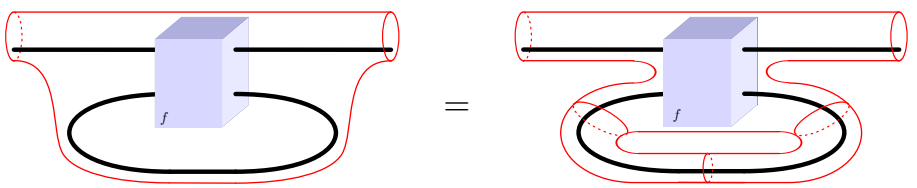

Proof. The result follows from the way the monoidal trace is constructed via the coalgebraic trace in (Jacobs, 2010): first, the functor $Y+(-): \mathbb{C} \rightarrow \mathbb{C}$ has an initial algebra in $\mathbb{C}$ given by the copower $\mathbb{N} \cdot Y$ with algebra map $\alpha_{Y}=\left[\kappa_{0},\left[\kappa_{n+1}\right]_{n \in \mathbb{N}}\right]: Y+\mathbb{N} \cdot Y \stackrel{\cong}{\rightrightarrows} \mathbb{N} \cdot Y$. The functor $U \otimes-: \mathbb{C} \rightarrow \mathbb{C}$ preserves coproducts by assumption, so the canonical map $d=\left[\mathrm{id} \otimes \kappa_{n}\right]_{n \in \mathbb{N}}: \mathbb{N} \cdot(U \otimes Y) \rightarrow U \otimes(\mathbb{N} \cdot Y)$ is an isomorphism. It is then also an isomorphism of initial algebras.

The general trace theory from (Hasuo et al., 2007) now says that $\mathbb{N} \cdot Y$ is the final coalgebra in the Kleisli category $\mathcal{K} \ell(T)$ of the functor $T(Y+(-))$. For a map $f: X+C \rightarrow$ $T(Y+C)$ we first take $\widehat{f}=T\left(\mathrm{id}+\kappa_{2}\right) \circ f: X+C \rightarrow Y+(X+C)$. It yields a unique map beh $(\widehat{f}): X+C \rightarrow T(\mathbb{N} \cdot Y)$ to the final coalgebra, and finally the trace map itself as $\operatorname{Tr}^{\mathcal{K} \ell}(f)=T(\nabla) \circ \operatorname{beh}(\widehat{f}) \circ \kappa_{1}: X \rightarrow T(Y)$.

We can similarly obtain a trace map $\operatorname{Tr}^{\mathcal{K} \ell}\left(f_{U}\right): U \otimes X \rightarrow U \otimes Y$ for the morphism $f_{U}=T\left(\right.$ dis $\left.^{-1}\right) \circ(U \otimes f) \circ$ dis: $U \otimes X+U \otimes C \rightarrow T(U \otimes Y+U \otimes X)$ used in Proposition that we are proving. We are done if the following diagram commutes.

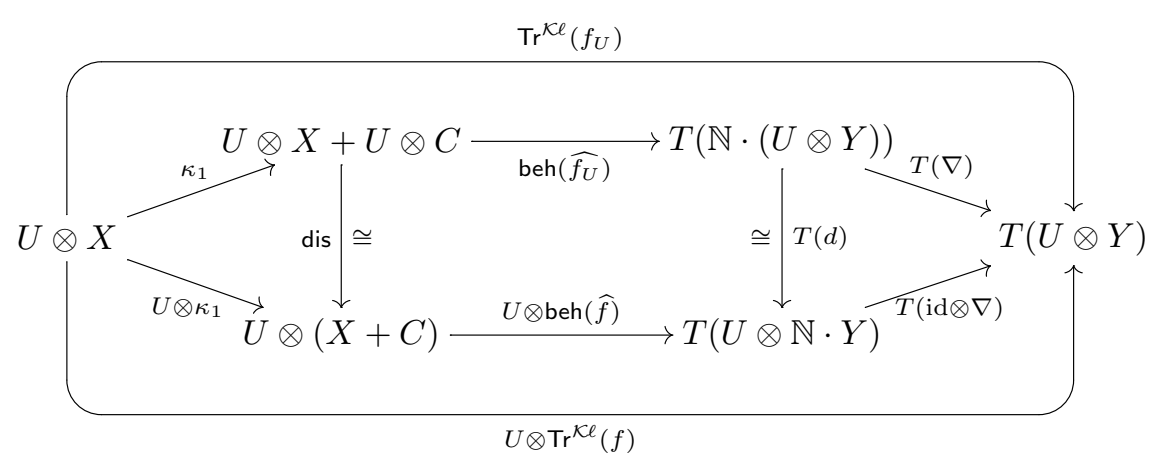

It is obvious that the two triangles commute. Commutation of the inner rectangle follows by a finality argument, in the Kleisli category:

$$
\begin{aligned}
& U \otimes Y+(U \otimes X+U \otimes Y) \Longrightarrow U \otimes Y+U \otimes \mathbb{N} \cdot Y \\
& \mathrm{id}+\kappa_{2} \uparrow \quad \cong \uparrow \mathrm{dis}^{-1} \\
& U \otimes Y+U \otimes C \quad U \otimes(Y+\mathbb{N} \cdot Y)
\end{aligned}
$$

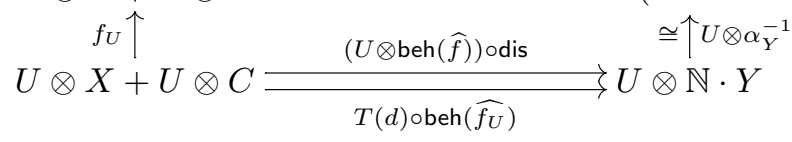

Remaining details are left to the reader. 
We now return to our framework of coalgebraic components, and wish to show how trace and state extension interact.

Lemma 6.5. Trace commutes with state extension:

$$
U \mid \operatorname{Tr}(c)=\operatorname{Tr}(U \mid c) \text { and } \operatorname{Tr}(c) \mid V=\operatorname{Tr}(c \mid V) .
$$

Proof. We use several standard properties of the trace $\operatorname{Tr}^{\mathcal{K} \ell}$ in the Kleisli category $\mathcal{K} \ell(T)$, such as (di)naturality, but we mainly depend on Proposition 6.4. We calculate in this Kleisli category:

$$
\begin{aligned}
& U \mid \operatorname{Tr}(c) \\
& =\alpha \bullet(U \otimes \operatorname{Tr}(c)) \bullet \alpha^{-1} \\
& \left.\left.=\alpha \bullet\left(U \otimes \operatorname{Tr}^{K \ell}\left(\operatorname{dis}^{-1} \bullet c \bullet \operatorname{dis}\right)\right) \bullet \operatorname{dis}\right)\right) \bullet \alpha^{-1} \\
& =\alpha \bullet \operatorname{Tr}^{K \ell}\left(\text { dis }^{-1} \bullet U \otimes\left(\text { dis }^{-1} \bullet c \bullet \text { dis }\right) \bullet \text { dis }\right) \bullet \alpha^{-1} \\
& \text { by Proposition } 6.4 \\
& =\operatorname{Tr}^{\mathcal{K} \ell}\left((\alpha+\mathrm{id}) \bullet \mathrm{dis}^{-1} \bullet\left(U \otimes \mathrm{dis}^{-1}\right) \bullet(U \otimes c) \bullet(U \otimes \mathrm{dis}) \bullet \operatorname{dis} \bullet\left(\alpha^{-1}+\mathrm{id}\right)\right) \\
& \text { by naturality of dis, see Lemma } 2.1 .1 \\
& =\operatorname{Tr}^{\mathcal{K} \ell}\left(\left(\mathrm{id}+\alpha^{-1}\right) \bullet(\mathrm{id}+\alpha) \bullet(\alpha+\mathrm{id}) \bullet\right. \\
& \left.\operatorname{dis}^{-1} \bullet\left(U \otimes \operatorname{dis}^{-1}\right) \bullet(U \otimes c) \bullet(U \otimes \operatorname{dis}) \bullet \operatorname{dis} \bullet\left(\alpha^{-1}+\mathrm{id}\right)\right) \\
& =\operatorname{Tr}^{\mathcal{K}}\left((\alpha+\alpha) \bullet \operatorname{dis}^{-1} \bullet\left(U \otimes \operatorname{dis}^{-1}\right) \bullet(U \otimes c) \bullet(U \otimes \operatorname{dis}) \bullet \operatorname{dis} \bullet\left(\alpha^{-1}+\alpha^{-1}\right)\right) \\
& \text { by dinaturality of } \operatorname{Tr}^{K \ell} \\
& =\operatorname{Tr}^{\mathcal{K} \ell}\left(\operatorname{dis}^{-1} \bullet \alpha \bullet(U \otimes c) \bullet \alpha^{-1} \bullet \text { dis }\right) \\
& \text { by Lemma } 2.1 .2 \\
& =\operatorname{Tr}^{\mathcal{K} \ell}\left(\operatorname{dis}^{-1} \bullet(U \mid c) \bullet \operatorname{dis}\right) \\
& =\operatorname{Tr}(U \mid c) \text {. }
\end{aligned}
$$

We immediately use this property in:

$$
\begin{aligned}
\operatorname{Tr}(c) \mid V & =(\gamma \otimes \mathrm{id}) \bullet(V \mid \operatorname{Tr}(c)) \bullet(\gamma \otimes \mathrm{id}) & & \\
& =(\gamma \otimes \mathrm{id}) \bullet \operatorname{Tr}(V \mid c) \bullet(\gamma \otimes \mathrm{id}) & & \text { as just proved } \\
& =\operatorname{Tr}((\gamma \otimes \mathrm{id}) \bullet(V \mid c) \bullet(\gamma \otimes \mathrm{id})) & & \text { by Lemma } 6.3 \\
& =\operatorname{Tr}(c \mid V) . & &
\end{aligned}
$$

\subsection{Trace and additive parallel composition}

The following lemma describes the interaction of trace and additive parallel composition. It will be crucial for proving the (di)naturality properties for $\operatorname{Tr}$ in the next subsection, which involve composition $\gg$ of components instead of Kleisli composition •

Lemma 6.6. For appropriately typed components $c, d$ one has:

$1 \operatorname{Tr}((d \square \operatorname{arr}(\mathrm{id})) \bullet c)=(d \mid I) \bullet \operatorname{Tr}(c) ;$ 
$2 \operatorname{Tr}(c \bullet(d \square \operatorname{arr}(\mathrm{id})))=\operatorname{Tr}(c) \bullet(d \mid I)$.

$3 \operatorname{Tr}((\operatorname{arr}(\mathrm{id}) \square d) \bullet c)=\operatorname{Tr}(c \bullet(\operatorname{arr}(\mathrm{id}) \square d))$.

Proof. We shall do the first and third point; the second works like the first.

$$
\begin{aligned}
\operatorname{Tr}((d \square \operatorname{arr}(\mathrm{id})) \bullet c) & =\operatorname{Tr}^{K \ell}\left(\operatorname{dis}^{-1} \bullet \operatorname{dis} \bullet(d|I+Y| \operatorname{arr}(\mathrm{id})) \bullet \operatorname{dis}^{-1} \bullet c \bullet \operatorname{dis}\right) \\
& =\operatorname{Tr}^{K \ell}\left((d \mid I+\mathrm{id}) \bullet \operatorname{dis}^{-1} \bullet c \bullet \operatorname{dis}\right) \\
& =(d \mid I) \bullet \operatorname{Tr}^{K \ell}\left(\operatorname{dis}^{-1} \bullet c \bullet \text { dis }\right) \quad \text { by naturality of } \operatorname{Tr}^{K \ell} \\
& =(d \mid I) \bullet \operatorname{Tr}(c) .
\end{aligned}
$$

And:

$$
\begin{aligned}
& \operatorname{Tr}((\operatorname{arr}(\mathrm{id}) \square d) \bullet c) \\
& \quad=\operatorname{Tr} \mathcal{K l}\left(\mathrm{dis}^{-1} \bullet \operatorname{dis} \bullet(\operatorname{arr}(\mathrm{id})|Y+I| d) \bullet \mathrm{dis}^{-1} \bullet c \bullet \mathrm{dis}^{-1}\right) \\
& \quad=\operatorname{Tr} \mathcal{K l}\left((\mathrm{id}+I \mid d) \bullet \mathrm{dis}^{-1} \bullet c \bullet \mathrm{dis}^{-1}\right) \\
& \quad=\operatorname{Tr} \mathcal{K l}\left(\operatorname{dis}^{-1} \bullet c \bullet \mathrm{dis}^{-1} \bullet(\mathrm{id}+I \mid d)\right) \quad \text { by dinaturality of } \operatorname{Tr}^{\mathcal{K}} \\
& \quad=\operatorname{Tr} \mathcal{K l}\left(\operatorname{dis}^{-1} \bullet c \bullet \mathrm{dis}^{-1} \bullet(\operatorname{arr}(\mathrm{id})|Y+I| d) \bullet \mathrm{dis}^{-1} \bullet \mathrm{dis}\right) \\
& \quad=\operatorname{Tr}(c \bullet(\operatorname{arr}(\mathrm{id}) \square d)) .
\end{aligned}
$$

\subsection{Trace axioms}

In the remainder of this section the trace axioms from (Joyal et al., 1996), formulated in a component setting (with explicit isomorphisms), are verified. For each axiom, drawing a pictorial (pseudo-)proof with tube diagrams is helpful in coming up with a rigorous, calculational proof. We will present such a pictorial proof for an exemplary case, namely the Post-Composition Naturality property.

\section{Yanking}

In the language of components the Yanking property can be formulated as a diagram of the form:

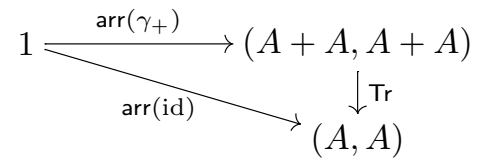

where $\gamma_{+}: A+A \stackrel{\cong}{\longrightarrow} A+A$ is the monoidal swap map associated with coproducts + , see Lemma 2.2.2. Pictorially:

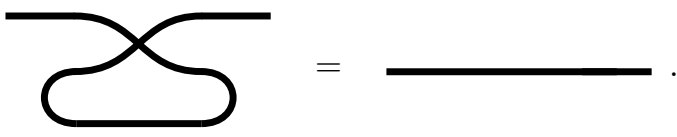

It has to be shown that the Kleisli trace of the following map

$$
I \otimes A+I \otimes A \stackrel{\text { dis }}{\cong} I \otimes(A+A) \stackrel{\operatorname{arr}\left(\gamma_{+}\right)}{\longrightarrow} T(I \otimes(A+A)) \stackrel{T\left(\mathrm{dis}^{-1}\right)}{\cong} T(I \otimes A+I \otimes A)
$$


is the (Kleisli) identity $\eta=\operatorname{arr}(\mathrm{id})$. This, together with the yanking property for the Kleisli trace operator $\mathrm{Tr}^{\mathcal{K} \ell}$, will be used in:

$$
\begin{aligned}
& \operatorname{Tr}(\operatorname{arr}(\gamma))=\operatorname{Tr}^{\mathcal{K} \ell}\left(T\left(\mathrm{dis}^{-1}\right) \circ \operatorname{arr}\left(\gamma_{+}\right) \circ \mathrm{dis}\right) \\
& \left.=\operatorname{Tr}^{\mathcal{K} \ell}\left(T\left(\mathrm{dis}^{-1}\right) \circ \eta \circ\left(\mathrm{id} \otimes \gamma_{+}\right) \circ \mathrm{dis}\right]\right) \\
& \stackrel{2.2 .2}{=} \operatorname{Tr}^{K \ell}\left(\eta \circ \mathrm{dis}^{-1} \circ \operatorname{dis} \circ \gamma_{+}\right) \\
& =\operatorname{Tr}^{\mathcal{K} \ell}\left(\eta \circ \gamma_{+}\right)=\operatorname{Tr}^{\mathcal{K} \ell}\left(\gamma_{+}^{\mathcal{K} \ell}\right)=\operatorname{id}_{I \otimes A}^{\mathcal{K} \ell}=\eta_{I \otimes A}=\operatorname{arr}\left(\operatorname{id}_{A}\right) \text {. }
\end{aligned}
$$

Post-composition naturality / tightening

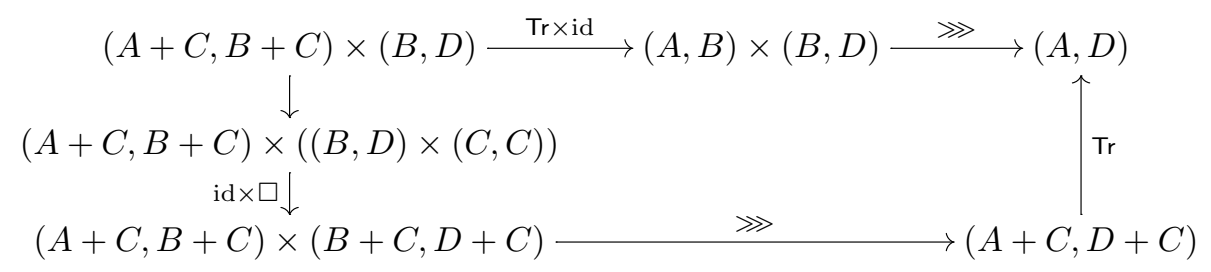

The usual string representation of this axiom is

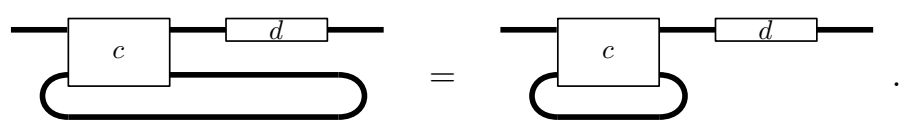

The aim is to prove for

$$
X \otimes(A+C) \stackrel{c}{\longrightarrow} T(X \otimes(B+C)) \quad \text { and } \quad Y \otimes B \stackrel{d}{\longrightarrow} T(Y \otimes D)
$$

that the following diagram commutes.

$$
\begin{array}{r}
T((X \otimes(Y \otimes I)) \otimes D) \frac{T((\mathrm{id} \otimes \rho) \otimes \mathrm{id})}{\cong} T((X \otimes Y) \otimes D) \\
\operatorname{Tr}(c \gg(d \square \operatorname{arr}(\mathrm{id}))) \uparrow \\
(X \otimes(Y \otimes I)) \otimes A \stackrel{\uparrow \operatorname{Tr}(c) \gg d}{\cong} \underset{(\mathrm{id} \otimes \rho) \otimes \mathrm{id}}{\cong}(X \otimes Y) \otimes A
\end{array}
$$


We shall make crucial use of Lemma 6.6 ; the rest is mainly bookkeeping.

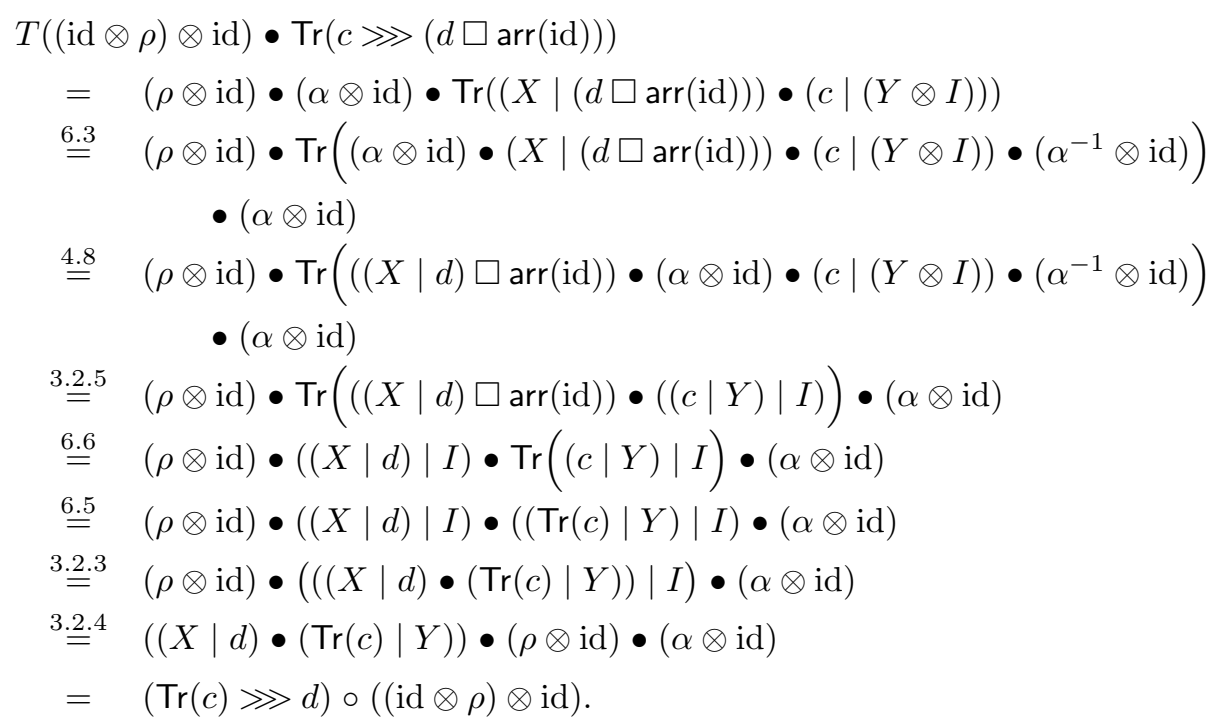

A pictorial (pseudo-)proof of the property is found in Figure 1. Although Lemmas 6.3, 6.5 and 6.6 are useful in the above calculational proof, more basic properties such as Lemma 5.1on which Lemmas 6.3, 6.5 and 6.6 rely-have clearer pictorial meanings. Therefore the latter are used in the pictorial proof.

Pre-composition naturality / tightening

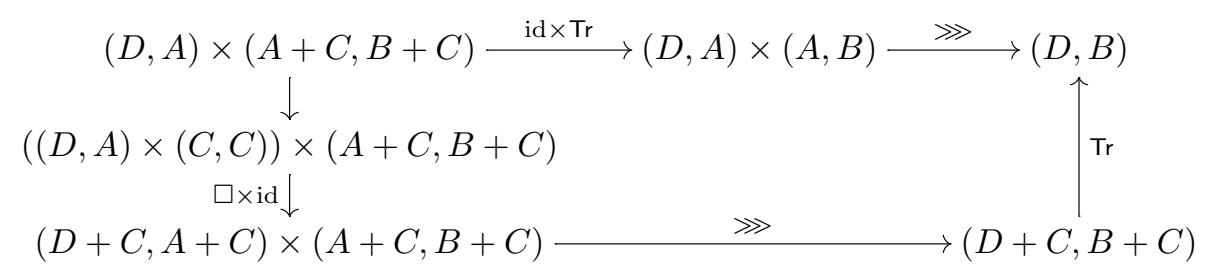

The aim is to prove for

$$
X \otimes(A+C) \stackrel{c}{\longrightarrow} T(X \otimes(B+C)) \quad \text { and } \quad Y \otimes D \stackrel{d}{\longrightarrow} T(Y \otimes A)
$$

that the following diagram commutes.

$$
\begin{array}{r}
T(((Y \otimes I) \otimes X) \otimes B) \frac{T((\rho \otimes \mathrm{id}) \otimes \mathrm{id})}{\cong} T((Y \otimes X) \otimes B) \\
\operatorname{Tr}((d \square \operatorname{arr}(\mathrm{id})) \ggg c) \uparrow \\
((Y \otimes I) \otimes X)) \otimes A \underset{(\rho \otimes \mathrm{id}) \otimes \mathrm{id}}{\cong}(Y \otimes X) \otimes A
\end{array}
$$

This is left to the interested reader. 


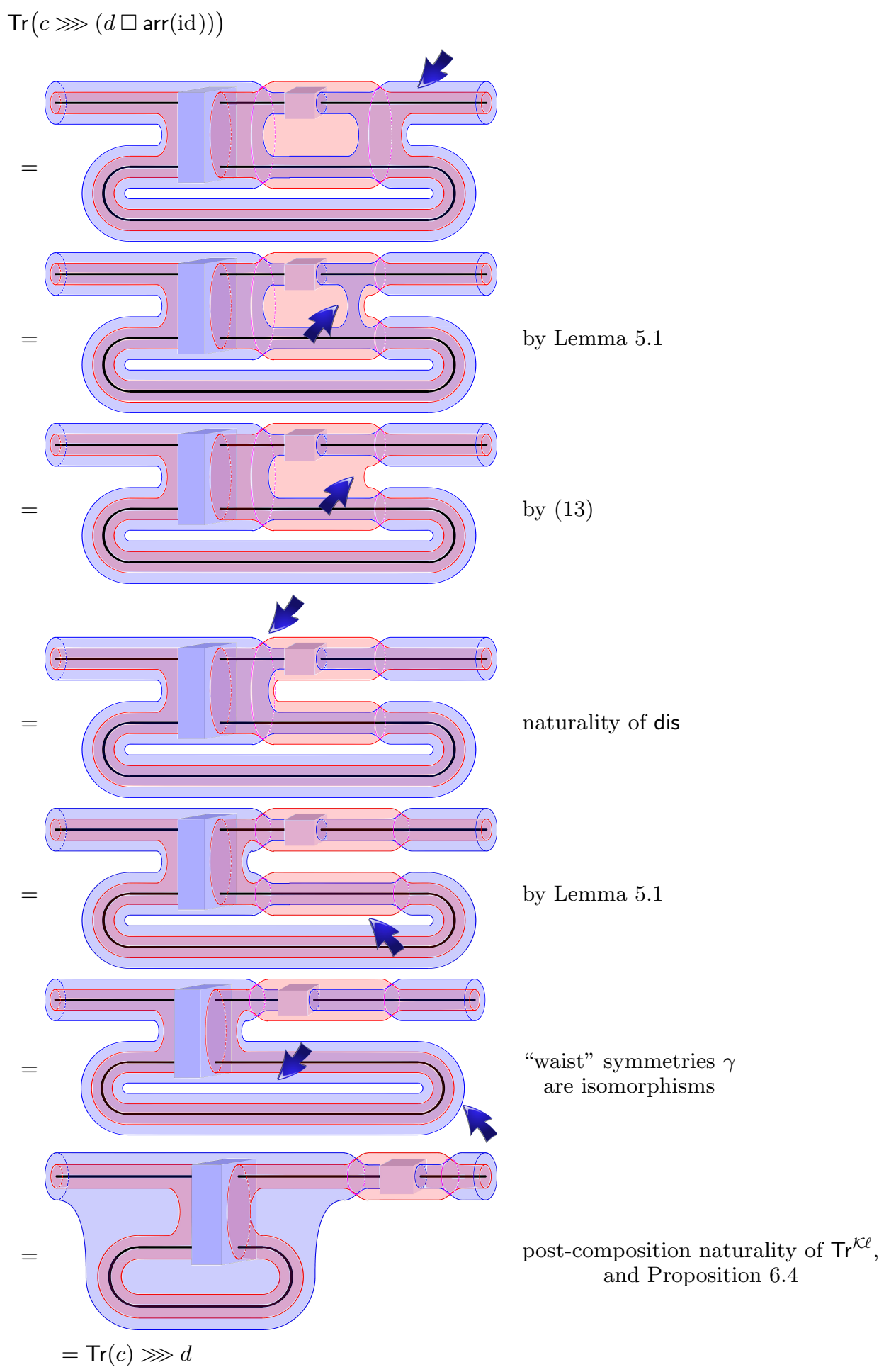

Figure 1. A pictorial proof of post-composition naturality. The pointers designate where transformation is going to occur. 


\section{Dinaturality}

$$
\begin{aligned}
& (A+C, B+D) \times\left((B, B) \times \frac{(A+C, B}{\times(D, C))^{\mathrm{id} \times \square}}+D\right) \times(B+\underbrace{D, B+C}_{\ggg}) \\
& \begin{array}{r}
\uparrow+C, B+D) \times((B, B) \times(D, C)) \\
\downarrow
\end{array} \\
& (A+C, B+D) \times(D, C) \\
& (A, B) \\
& (D, C) \times(A+C, B+D) \\
& \downarrow \\
& ((A, A) \times(D, C)) \times(A+C, B+D)) \underset{\square \times \mathrm{id}}{(A+D}, A+C) \times(A+\stackrel{\overbrace{C, B+D)}}{\gg}(A+D, B+D)
\end{aligned}
$$

In terms of string diagrams:

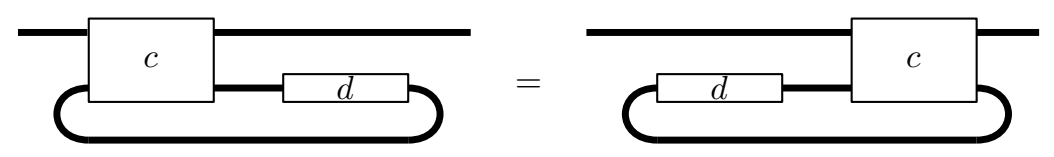

For coalgebras

$$
X \otimes(A+C) \stackrel{c}{\longrightarrow} T(X \otimes(B+D)) \quad \text { and } \quad Y \otimes D \stackrel{d}{\longrightarrow} T(Y \otimes C)
$$

we show that the following diagram commutes.

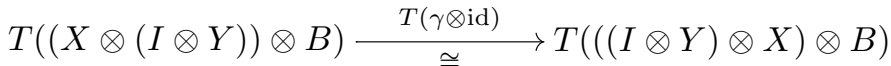

$$
\begin{aligned}
& \operatorname{Tr}\left(c \gg\left(\operatorname{arr}\left(\operatorname{id}_{B}\right) \square d\right) \uparrow \quad \cong \quad \uparrow \operatorname{Tr}\left(\left(\operatorname{arr}\left(\operatorname{id}_{A}\right) \square d\right) \ggg c\right)\right. \\
& (X \otimes(I \otimes Y)) \otimes A \underset{T(\gamma \otimes \mathrm{id})}{\cong}((I \otimes Y) \otimes X) \otimes A
\end{aligned}
$$


The essence is again in Lemma 6.6, but with quite some bookkeeping this time.

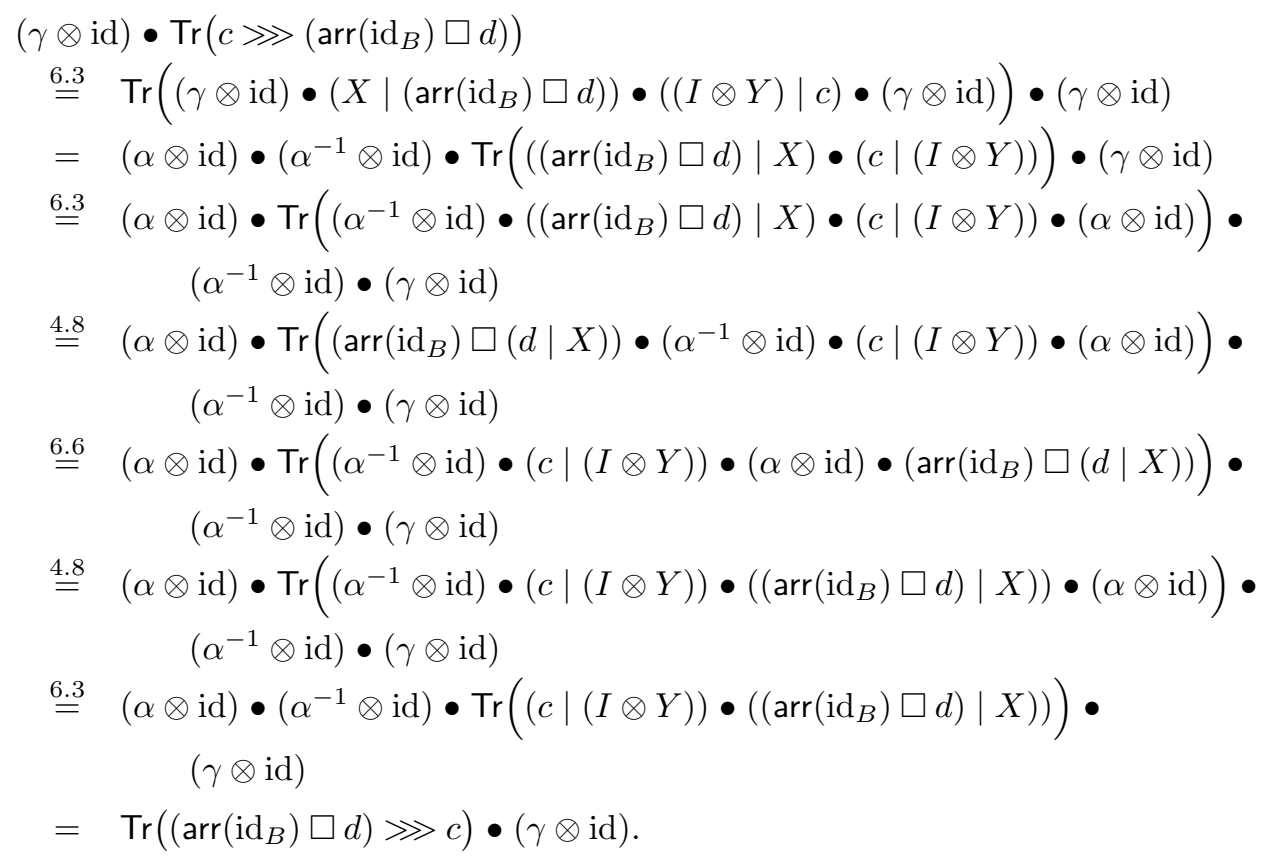

\section{Unit vanishing}

The relevant component diagram is:

$$
\begin{aligned}
& (A+0, B+0) \longrightarrow(A, B) \\
& \left\langle\operatorname{arr}\left(\rho_{+}^{-1}\right), \operatorname{id}, \operatorname{arr}\left(\rho_{+}\right)\right\rangle \downarrow \quad \uparrow \ggg \\
& (A, A+0) \times(A+0, B+0) \times(B+0, B) \underset{\ggg \times \mathrm{id}}{\longrightarrow}(A, B+0) \times(B+0, B)
\end{aligned}
$$

where we have to keep in mind that the $\rho_{+}: C+0 \stackrel{\cong}{\longrightarrow} C$ refers to the monoidal isomorphism wrt. the coproducts + on $\mathbb{C}$.

Pictorially, the axiom asserts

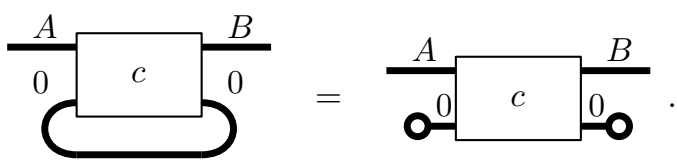

We will prove that there is an isomorphism of components:

$$
\begin{aligned}
& T(((I \otimes X) \otimes I) \otimes B) \underset{T((\lambda \circ \rho) \otimes \mathrm{id})}{\cong} T(X \otimes B) \\
& \left(\operatorname{arr}\left(\rho_{+}^{-1}\right) \ggg c\right) \ggg \operatorname{arr}\left(\rho_{+}\right) \uparrow \quad \uparrow \quad \uparrow \operatorname{Tr}(c) \\
& ((I \otimes X) \otimes I) \otimes A \underset{(\lambda \circ \rho) \otimes \mathrm{id}}{\cong} X \otimes A
\end{aligned}
$$


The heart of the matter is:

$$
\begin{array}{rlrl}
\operatorname{Tr} & (c) & & \\
& =\operatorname{Tr}^{\mathcal{K} \ell}\left(\operatorname{dis}^{-1} \bullet c \bullet \text { dis }\right) & \text { with dis: }(X \otimes A)+(X \otimes 0) \cong X \otimes(A+0) \\
=\rho_{+} \bullet \operatorname{dis}^{-1} \bullet c \bullet \operatorname{dis} \bullet \rho_{+}^{-1} & \text { by vanishing for } \operatorname{Tr}^{\mathcal{K} \ell}, \text { since } X \otimes 0 \text { is initial } \\
=\left(\operatorname{id} \otimes \rho_{+}^{-1}\right) \bullet c \bullet\left(\operatorname{id} \otimes \rho_{+}\right) & \text {by Lemma } 2.2 .1 .
\end{array}
$$

Hence we obtain the required isomorphism of components:

$$
\begin{array}{cl}
((\lambda \circ \rho) \otimes \mathrm{id}) \bullet\left(\left(\operatorname{arr}\left(\rho_{+}^{-1}\right) \gg c\right) \gg \operatorname{arr}\left(\rho_{+}\right)\right) \\
\stackrel{4.2 .2}{=}(\lambda \otimes \mathrm{id}) \bullet\left(\mathrm{id} \otimes \rho_{+}\right) \bullet\left(\operatorname{arr}\left(\rho_{+}^{-1}\right) \gg c\right) \bullet(\rho \otimes \mathrm{id}) \\
=\quad\left(\mathrm{id} \otimes \rho_{+}\right) \bullet(\lambda \otimes \mathrm{id}) \bullet\left(\operatorname{arr}\left(\rho_{+}^{-1}\right) \gg c\right) \bullet(\rho \otimes \mathrm{id}) \\
\stackrel{4.2 .2}{=}\left(\mathrm{id} \otimes \rho_{+}\right) \bullet c \bullet\left(\mathrm{id} \otimes \rho_{+}^{-1}\right) \bullet(\lambda \otimes \mathrm{id}) \bullet(\rho \otimes \mathrm{id}) \\
=\operatorname{Tr}(c) \bullet((\lambda \circ \rho) \otimes \mathrm{id}), \quad \text { as shown above. }
\end{array}
$$

\section{Tensor vanishing}

Again we have to distinguish carefully between the monoidal associativity isomorphisms $\alpha_{+}$and $\alpha$, for coproduct + and tensor $\otimes$, respectively. The component diagram is:

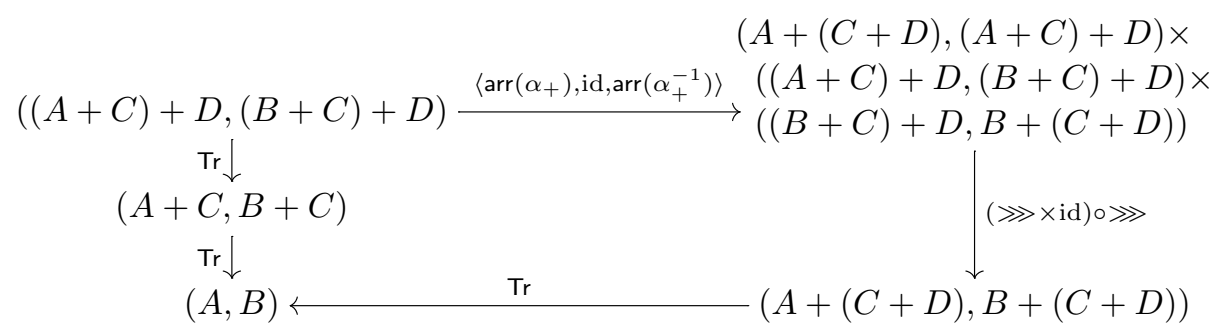

Pictorially,

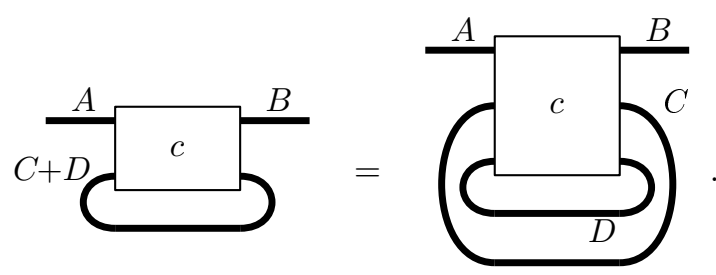

Our aim is to prove, for a component $X \otimes((A+C)+D) \stackrel{c}{\longrightarrow} X \otimes((B+C)+D)$, that we have an isomorphism of components:

$$
\begin{aligned}
& T(((I \otimes X) \otimes I) \otimes A) \stackrel{T((\lambda \circ \rho) \otimes \mathrm{id})}{\simeq} T(X \otimes A) \\
& \operatorname{Tr}\left(\left(\operatorname{arr}\left(\alpha_{+}\right) \ggg c\right) \ggg \operatorname{arr}\left(\alpha_{+}^{-1}\right)\right) \uparrow \quad \cong \quad \uparrow \operatorname{Tr}(\operatorname{Tr}(c)) \\
& ((I \otimes X) \otimes I) \otimes A \underset{(\lambda \circ \rho) \otimes \mathrm{id}}{\cong} X \otimes A
\end{aligned}
$$


This is done as follows.

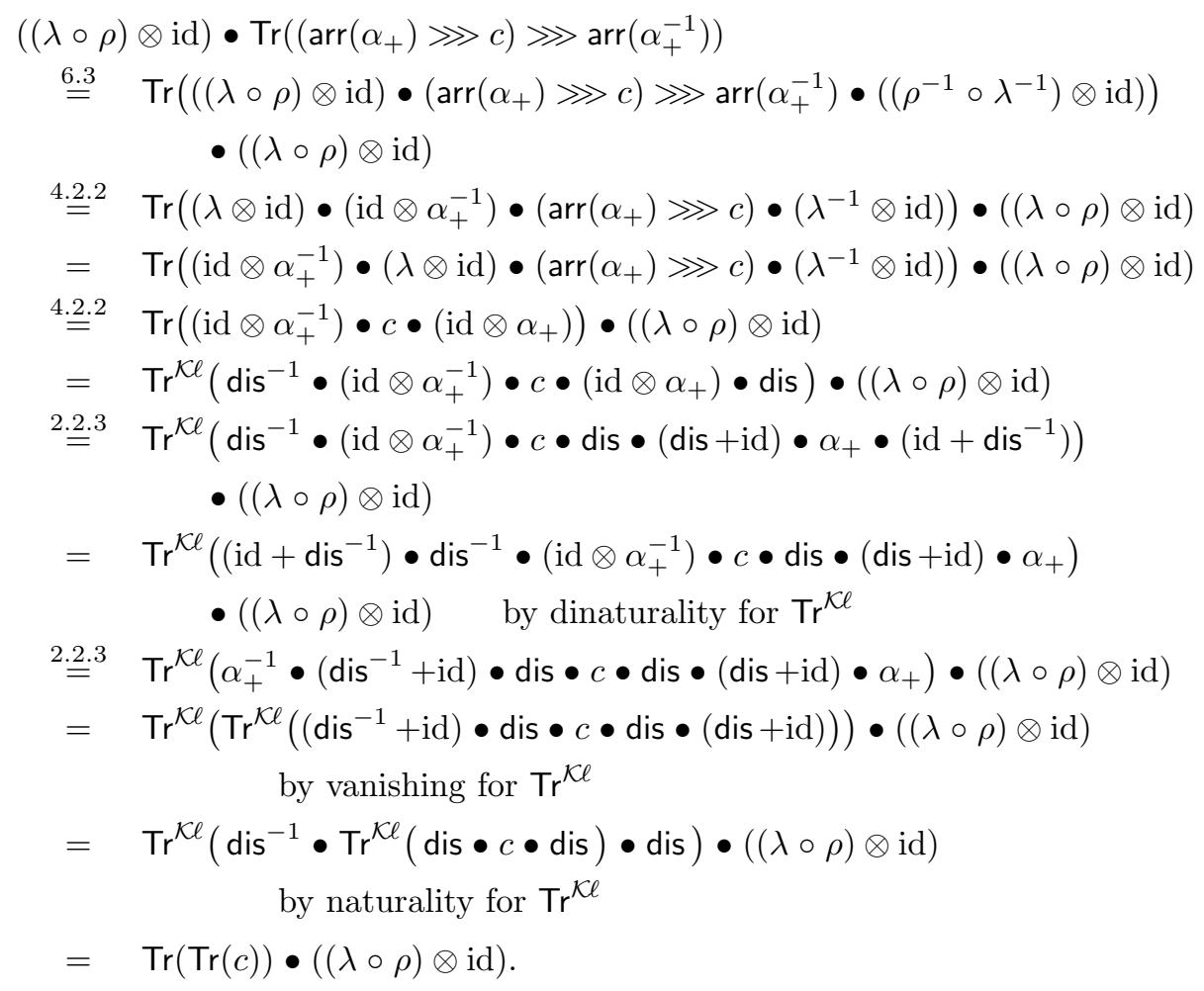

\section{Superposing}

The relevant diagram for components is:

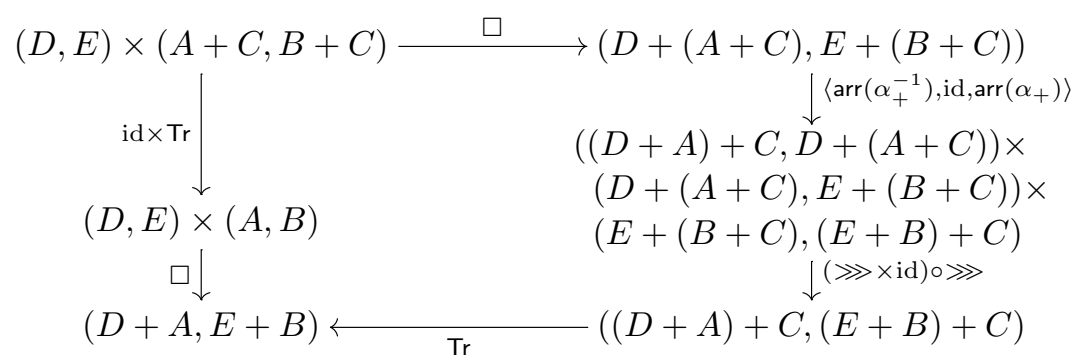

Pictorially,

For coalgebraic components

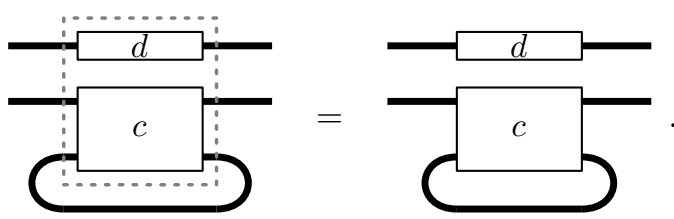

$$
Y \otimes E \stackrel{d}{\longrightarrow} Y \otimes D \text { and } X \otimes(A+C) \stackrel{c}{\longrightarrow} X \otimes(B+C)
$$


this involves an isomorphism of components:

$$
\begin{aligned}
& T(((I \otimes(Y \otimes X)) \otimes I) \otimes(E+B)) \stackrel{T((\lambda \circ \rho) \otimes \mathrm{id})}{\cong} T((Y \otimes X) \otimes(E+B)) \\
& \operatorname{Tr}\left(\left(\operatorname{arr}\left(\alpha_{+}^{-1}\right) \gg(d \square c)\right) \gg \operatorname{arr}\left(\alpha_{+}\right)\right) \uparrow \quad \uparrow d \square \operatorname{Tr}(c) \\
& ((I \otimes(Y \otimes X)) \otimes I) \otimes(E+B) \underset{(\lambda \circ \rho) \otimes \mathrm{id}}{\stackrel{\cong}{\longrightarrow}}(Y \otimes X) \otimes(E+B)
\end{aligned}
$$

One proceeds along by now familiar lines.

\section{Traced monoidal category of resumptions}

In this section we use the previous results - about operators and equations on componentsto prove that the category of T-resumptions is traced symmetric monoidal. This general result holds for a large class of monads $T$ with suitable assumptions, hence generalises the result in (Abramsky et al., 2002) that focuses on the lift monad $T=1+(-)$. Although we do not fully expose it, the technical development is an instance of the theory - developed previously in (Hasuo et al., 2008; Hasuo et al., 2009) — on the microcosm principle (Baez and Dolan, 1998). Application of this general theory exploits characterisation of resumptions as elements of a final component.

Throughout the current section the base category $\mathbb{C}$ is fixed to be Set, the category of sets and functions. It is a symmetric monoidal closed category with Cartesian product $\times$ as tensor $\otimes$ and the singleton 1 as monoidal unit $I$; it is also equipped with distributive coproducts,+ 0 . All the results in the previous sections are valid in this base category.

\subsection{Resumptions}

The notion of resumption has been introduced in (Milner, 1975) for the purpose of providing denotational semantics for interactive computing agents. A historical account is found in (Abramsky et al., 2002, Section 5.4.1); our recap here of the notion is adapted for the current context.

First let us think of a component $X \times A \stackrel{c}{\rightarrow} X \times B$, a map in Set. It is a component (3) where $T=$ id is the trivial monad and $\otimes$ is chosen to be Cartesian product $\times$. It belongs to the category $\mathbf{C o m p}(\mathrm{id}, A, B)$; with $T=$ id this component does not exhibit any effect in its execution. In the theory of automata, such a machine is called a Mealy machine; it can also be thought of as a (simple version of a) transducer. The task that such a machine is expected to perform is transformation of (infinite) $A$-streams into $B$-streams; the transformation should be performed in a letter-by-letter manner.

A resumption is an extensional view of the behaviour of such a machine. Specifically, the above machine $c$ induces a resumption formalised as a stream function $r: A^{\omega} \rightarrow B^{\omega}$ which is causal, meaning that the $n$-th letter of the output stream only depends on the first $n$ letters of the input. ${ }^{\S}$

\footnotetext{
$\S$ This is how they are formalised in (Rutten, 2006). Equivalent formulations are: as string functions $A^{*} \rightarrow B^{*}$ that are length-preserving and prefix-closed (Pattinson, 2003); and as functions $A^{+} \rightarrow B$ where $A^{+}$is the set of (finite-length) strings of length $\geq 1$.
} 
Let us now take a coalgebraic view on components and resumptions. A component $X \times A \stackrel{c}{\rightarrow} X \times B$ is the same thing as a map $X \rightarrow(X \times B)^{A}$ hence is a coalgebra for the functor $(-\times B)^{A}$. As is noted in (Abramsky et al., 2002), the "behaviours by coinduction" paradigm in the theory of coalgebra (Jacobs and Rutten, 1997; Rutten, 2000) is also valid in this setting. Namely, the set $Z_{A, B}$ of resumptions (i.e. causal stream functions) carries a canonical $(-\times B)^{A}$-coalgebra structure:

$$
\begin{gathered}
Z_{A, B} \stackrel{\zeta_{A, B}}{\cong}\left(Z_{A, B} \times B\right)^{A} \\
\left(r: A^{\omega} \rightarrow B^{\omega}, \text { causal }\right) \longmapsto \lambda a \cdot\left(\left(\lambda \overrightarrow{a^{\prime}} \cdot \operatorname{tail}\left(r\left(a \cdot \overrightarrow{a^{\prime}}\right)\right)\right), \operatorname{head}(r(a \cdot \vec{a}))\right) .
\end{gathered}
$$

Here $a \cdot \vec{a}$ is a letter $a \in A$ followed by an arbitrary stream $\vec{a}$; the value of head $(r(a \cdot \vec{a}))$ does not depend on $\vec{a}$ since $r$ is causal. Moreover, it is standard that this coalgebra $\zeta_{A, B}$ is a final one. Given an arbitrary component $c: X \times A \rightarrow X \times B$ (i.e. a coalgebra $\left.c: X \rightarrow(X \times B)^{A}\right)$ finality of $\zeta_{A, B}$ induces the behaviour map:

$$
\begin{gathered}
(X \times B)^{A}--\rightarrow\left(Z_{A, B} \times B\right)^{A} \\
c \uparrow \\
X--\overline{\operatorname{binal} \uparrow \cong} \cong-\rightarrow Z_{A, B}
\end{gathered}
$$

which carries a state $x \in X$ to the behaviour beh $(c)(x)$ of $c$, in case of execution with $x$ as the initial state, represented by a resumption. To summarise: the set of resumptions from $A$ to $B$ carries a final $(-\times B)^{A}$ coalgebra.

We have restricted ourselves to the trivial monad $T=\mathrm{id}$ for the purpose of illustration of resumptions. However this choice of a monad $T$ does not satisfy the assumption in Section 6 for $\mathcal{K} \ell(T)$ to be traced: an iteration of a total function can fail to be total because of an infinite loop. For monads $T$ in general - especially for those which satisfy the assumption in Section 6 - we generalise the above characterisation of resumptions as follows. The same has been done in (Abramsky et al., 2002).

Definition 7.1 ( $T$-resumption). Let $T$ be a monad on Set such that, for any sets $A$ and $B$, a final $(T(-\times B))^{A}$-coalgebra

$$
\zeta_{A, B}^{T}: Z_{A, B}^{T} \stackrel{\cong}{\longrightarrow}\left(T\left(Z_{A, B}^{T} \times B\right)\right)^{A} \quad \text { in Set }
$$

exists. A $T$-resumption from $A$ to $B$ is an element of the carrier $Z_{A, B}^{T}$.

Morphisms of $T(-\times B)^{A}$-coalgebras are precisely morphisms of components: there is an isomorphism of categories: $\operatorname{Coalg}\left(T(-\times B)^{A}\right) \cong \operatorname{Comp}(T, A, B)$. Hence $T$-resumptions form the state space of a final component.

Assumption 7.2. In the remainder of this section we assume that a monad $T$ on Set satisfies both the assumption in Section 6 (namely (Jacobs, 2010, Requirements 4.7)) as well as the one in Definition 7.1. The former consists of $\mathcal{K} \ell(T)$ being $\mathbf{D c p o}{ }_{\perp}$-enriched, $T$ being "semi-additive", etc.; it ensures that we have a trace operator $\operatorname{Tr}^{\mathcal{K} \ell}$. The latter enables us to capture resumptions by a final coalgebra.

Such monads include: the lift monad $1+(-)$; the $\kappa$-bounded powerset monad $\mathcal{P}^{<\kappa}$ with 
an uncountable weakly inaccessible cardinal $\kappa$; and the (discrete) subdistribution monad $\mathcal{D}$. Regarding the monad $\mathcal{P}<\kappa$, the cardinal $\kappa$ must be larger than $\aleph_{0}$ so that an increasing $\omega$-sequence in the set $\mathcal{P}^{<\kappa}(X)$ has its supremum inside $\mathcal{P}^{<\kappa}(X)$; this is needed for the trace assumption in Section 6. At the same time $\kappa$ is assumed to be weakly inaccessible so that Barr's final coalgebra theorem (Barr, 1993) ensures existence of final coalgebras. Such explicit size restriction is not needed for the subdistribution monad $\mathcal{D}$, since the condition $\sum_{x} d(x) \leq 1$ implies that the support $\{x \in X \mid d(x) \neq 0\}$ is at most countable. See e.g. (Sokolova, 2005, Proposition 2.1.2).

It is generally hard to concretely describe what a $T$-resumption looks like. It is a treemuch like a synchronisation tree (Milner, 1980) — but its depth and branching degree are very often larger than $\aleph_{0}$. A tractable description is possible for the lift monad: much like for the identity monad, it is represented by a function $r: A^{\omega} \rightarrow B^{*}+B^{\omega}$ with a suitably generalised causality requirement.

\subsection{The microcosm principle}

One can form the category of $T$-resumptions by arranging $T$-resumptions as morphisms in the category.

Definition 7.3 (The category $T$-Res). For a monad $T$ satisfying Assumption 7.2, we define the category of $T$-resumptions, denoted by $T$-Res, by the following data.

- An object $A$ of $T$-Res is a set $A \in$ Set.

- An arrow $r: A \rightarrow B$ in $T$-Res is a $T$-resumption from $A$ to $B$ (cf. Definition 7.1). Thus we have: $\operatorname{Hom}_{T-\operatorname{Res}}(A, B)=Z_{A, B}^{T}$.

Its actual structure as a category - composition and identity - shall be described in short.

The main point of (Abramsky et al., 2002, Section 5.4) is that the category of resumptions $(1+(-))$-Res for the lift monad is symmetric traced monoidal, and that it gives rise to a compact closed category of (stateful) games (Abramsky and Jagadeesan, 1994) after applying the Int-construction (Joyal et al., 1996). Its generalisation to a wider class of monads $T$ (other than $T=1+(-)$ ) is one of our main technical contribution.

Theorem 7.4. For a monad $T$ satisfying Assumption 7.2, the category $T$-Res of $T$ resumptions is symmetric traced monoidal.

This result, in fact, is an immediate corollary of what we have observed: the traced monoidal structure of $T$-Res follows from the composition and trace operators for components introduced in Section 4 and Section 6.

In order to illustrate the situation, let us think of arranging components - instead of resumptions - as morphisms from $A$ to $B$. Between such components $c$ and $d$ with the same input/output types we possibly have a morphism of components, see (4). The latter "morphism" $f$ can be drawn between components, as follows.

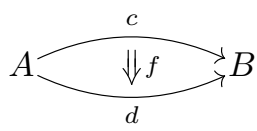


This motivates a 2-categorical approach to components. The envisaged 2-category has sets as 0-cells, components as 1-cells and morphisms of components as 2-cells. Naturally one would introduce (horizontal) composition of 1-cells by the sequential composition $\gg$ of components (Section 4); and the identity 1-cell $A \rightarrow A$ by the one-state component $\operatorname{arr}\left(\operatorname{id}_{A}\right)$ from (5).

However, Lemma 4.2.2-3 indicates that such horizontal composition of 1-cells satisfies the unit law and associativity only up-to canonical isomorphisms. This makes the envisaged structure not a 2-category, but a bicategory; see e.g. (Borceux, 1994). The resulting bicategory is much like the one in the bicategorical approach to processes (Katis et al., 1997). Let us denote the bicategory by $\operatorname{Comp}(T)$. This extends our previous notation, since its hom-category from $A$ to $B$ is given by the category $\operatorname{Comp}(T, A, B)$ of components.

What we have shown in Section 6 is essentially that the bicategory $\operatorname{Comp}(T)$ is equipped with traced monoidal structure. Its underlying monoidal structure is given by additive parallel composition $\square$ (Section 4.2); in particular it is binary coproduct + on objects.

The way we look at the category $T$-Res of resumptions is as a "thin slice" of the bicategory $\operatorname{Comp}(T)$ of components. The two have the same family of objects; the former's homset $T$ - $\operatorname{Res}(A, B)$ resides in the latter's $\operatorname{Comp}(T, A, B)$ as (the carrier of) a 1-cell; still $T-\operatorname{Res}(A, B)$ is "behaviourally universal" via its finality.

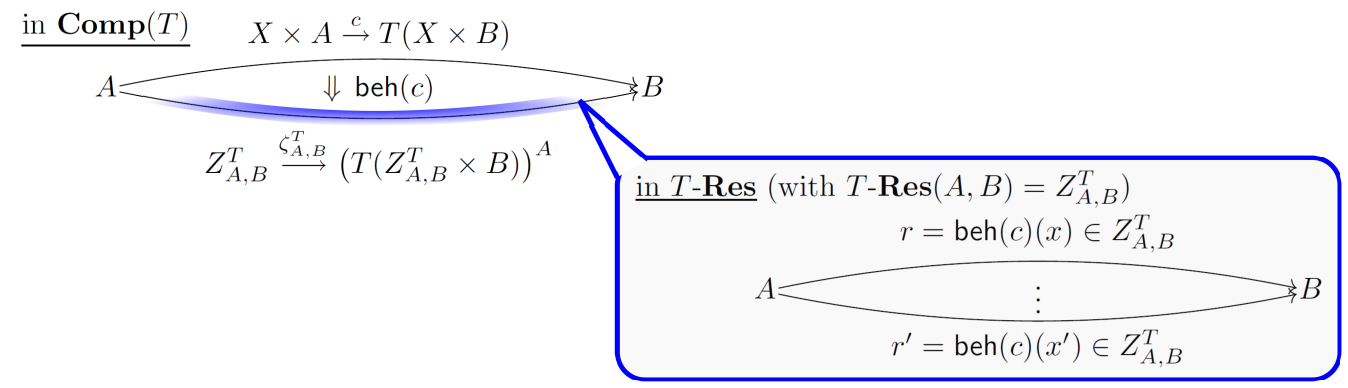

We then derive the structure of $T$-Res as a traced monoidal category, from the corresponding "outer" structure of $\operatorname{Comp}(T)$; it follows from the general theory developed previously in (Hasuo et al., 2008; Hasuo et al., 2009). The general theory identifies the situation as an instance of the microcosm principle (Baez and Dolan, 1998). The latter refers to a situation where "an algebra resides in another algebra, both for the same algebraic specification", a prototypical example of which is a monoid in a monoidal category (Mac Lane, 1998). As a result, the homsets of $T$-Res form a traced monoidal category, residing in the hom-categories of $\operatorname{Comp}(T)$ that form a "traced monoidal bicategory"; see (Hasuo et al., 2009) for the details of the generic situation. Rather than fully laying out the general theory, however, we shall now describe its concrete instantiation adapted to the current setting.

ฯ Axiomatisation of the notion of "traced monoidal bicategory" would involve delicate coherence conditions. We do not aim at such general Axiomatisation but focus on one specific instance. 


\subsection{Resumptions form a category}

Notation 7.5. The functor $(T(-\times B))^{A}$ - for which a coalgebra is a component with $A$-input and $B$-output - is denoted by $F_{A, B}$. The monad $T$ is fixed throughout the rest, and so it is suppressed. Therefore we write $Z_{A, B}$ rather than $Z_{A, B}^{T}$ for the homset of T-resumptions.

We first derive the sequential composition operator $\circ^{T-R e s}$ that acts on resumptions; it is obtained from how we compose arrows in $T$-Res. The following coinduction diagram in Set defines the operator:

$$
\begin{array}{r}
F_{A, C}\left(Z_{A, B} \times Z_{B, C}\right)---\rightarrow F_{A, C}\left(Z_{A, C}\right) \\
\zeta_{A, B} \gg \zeta_{B, C} \uparrow \\
Z_{A, B} \times Z_{B, C}--\underset{{ }_{A, B, C}^{T-R e s}}{-\overline{T i n a l} \uparrow \zeta_{A, C}}
\end{array}
$$

Recall that $\zeta_{A, B}$ is a final $F_{A, B}$-coalgebra (Definition 7.1). The sequential composition of $\zeta_{B, C}$ after $\zeta_{A, B}$ yields the component shown on the left, which is an $F_{A, C^{-c o a l g e b r a}}$ with a state space $Z_{A, B} \times Z_{B, C}$. It then induces a unique map into the final $F_{A, C}$-coalgebra, as in the above diagram. Thus we have obtained a function

$$
\begin{aligned}
\stackrel{\text { o }}{T \text {-Res }}: B, C & Z_{A, B} \times Z_{B, C} \longrightarrow Z_{C, A}, \quad \text { that is } \\
& \operatorname{Hom}_{T-\operatorname{Res}}(A, B) \times \operatorname{Hom}_{T-\operatorname{Res}}(B, C) \longrightarrow \operatorname{Hom}_{T-\operatorname{Res}}(A, C) .
\end{aligned}
$$

Similarly, the identity morphism $\operatorname{id}_{A}^{T-R e s}$ in $T$-Res is derived by coinduction, from the one-state component $\operatorname{arr}\left(\operatorname{id}_{A}\right)$.

$$
\begin{aligned}
& F_{A, A}(1)---\rightarrow F_{A, A}\left(Z_{A, A}\right)
\end{aligned}
$$

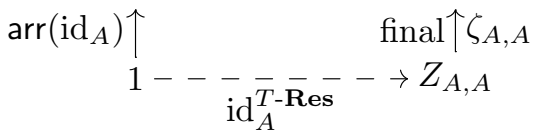

Let us prove associativity of $\circ^{T-R e s}$. The emphasis here is on the fact that, via coinduction, the goal is essentially reduced to associativity (Lemma 4.2.3) of $\gg$ - the corresponding "outer" operator.

Specifically, by diagram (22), the map $\circ_{A, B, C}^{T \text {-Res }}: Z_{A, B} \times Z_{B, C} \longrightarrow Z_{C, A}$ is a component morphism from $\zeta_{A, B} \gg \zeta_{B, C}$ to $\zeta_{A, C}$. Hence by the functoriality of $\gg$ (Lemma 4.2.4) we obtain a component morphism

$$
\circ_{A, B, C}^{T-\text {Res}} \times \operatorname{id}_{Z_{C, D}}:\left(\zeta_{A, B} \gg \zeta_{B, C}\right) \gg \zeta_{C, D} \longrightarrow \zeta_{A, C} \gg \zeta_{C, D} .
$$

This means that the left square in the following diagram commutes. The right one commutes, too, since it is the diagram $(22)$ defining ${ }_{0}^{T}$-Res,$D$.

$$
\begin{aligned}
& F_{A, D}\left(\left(Z_{A, B} \times Z_{B, C}\right) \times Z_{C, D}\right) \longrightarrow F_{A, D}\left(Z_{A, C} \times Z_{C, D}\right)-\rightarrow F_{A, D}\left(Z_{A, D}\right) \\
& \left(\zeta_{A, B} \gg \zeta_{B, C}\right) \gg \zeta_{C, D} \uparrow \quad \zeta_{A, C} \gg \zeta_{C, D} \uparrow \text { final } \uparrow \zeta_{A, D} \\
& \left(Z_{A, B} \times Z_{B, C}\right) \times Z_{C, D} \underset{\mathrm{o}_{A, B, C}^{T-\mathbf{R e s}} \times Z_{C, D}}{\longrightarrow} Z_{A, C} \times Z_{C, D}-\underset{{ }_{A, C, D}^{T-\mathbf{R e s}}}{-} \rightarrow Z_{A, D}
\end{aligned}
$$


The next diagram in Set commutes for the same reasons, in which the top square commutes by associativity of $\gg$ (Lemma 4.2.3).

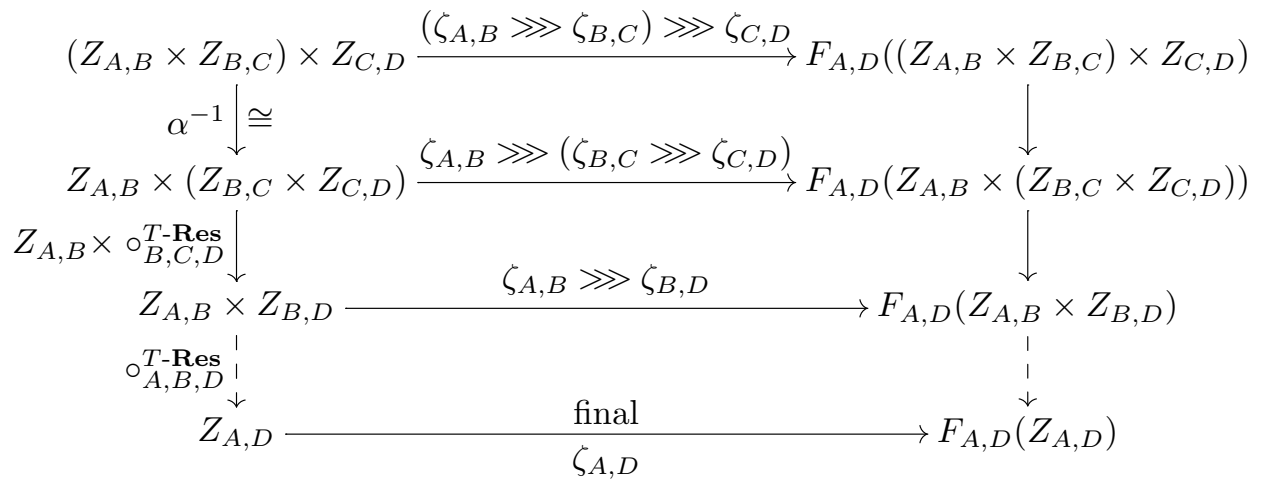

(In the last diagram we wrote coalgebras $X \rightarrow F X$ horizontally instead of vertically, unlike our convention elsewhere. This is purely for the typesetting reason.)

We conclude that the following diagram commutes, since the last two diagrams show that the two composites are both coalgebra morphisms from $\left(\zeta_{A, B} \gg \zeta_{B, C}\right) \gg \zeta_{C, D}$ to a final coalgebra $\zeta_{A, D}$.

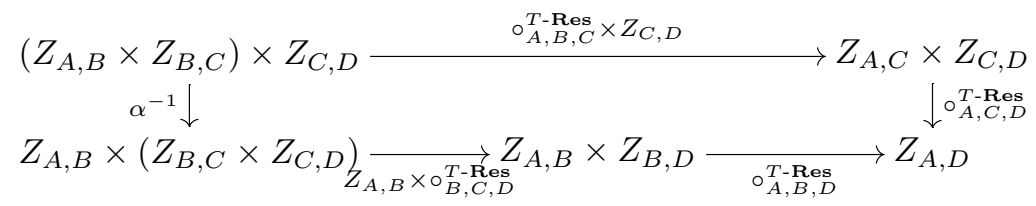

This is associativity of $\circ^{T-R e s}$.

The left- and right-unit laws for $T$-Res amount to the following diagram:

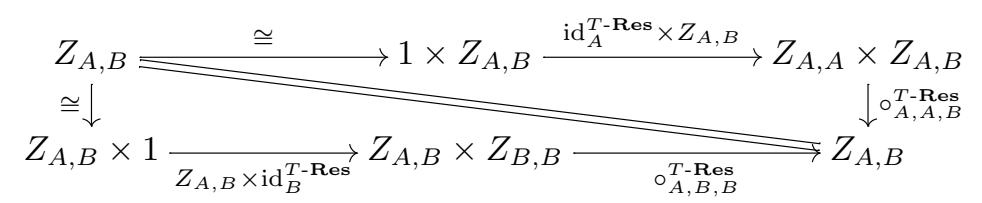

The diagram commutes essentially due to the unit laws (Lemma 4.2.2) for the outer operators $\gg$ and arr(id), much like for the associativity.

We summarise what we have so far.

Proposition 7.6. The data T-Res in Definition 7.3 indeed forms a category, with composition of arrows given by $\circ^{T \text {-Res }}$ in $(22)$ and identity arrows by $\mathrm{id}^{T-\boldsymbol{R} \text { es }}$ in $(23)$.

In fact the last proposition is a special case of (Krstić et al., 2001, Theorem 1). The latter result is more general because it works for an axiomatically introduced class of functors $\left\{F_{A, B}\right\}_{A, B}$-which roughly corresponds to the notion of lax $\mathbb{L}$-functor in (Hasuo et al., 2008; Hasuo et al., 2009) -instead of our concrete description $F_{A, B}=(T(B \times-))^{A}$ (Notation 7.5). From now on, however, we go beyond (Krstić et al., 2001) by introducing a symmetric monoidal structure on $T$-Res and a trace operator on top of it. 


\subsection{Resumptions carry symmetric monoidal structure}

Endowing the category $T$-Res with traced monoidal structure goes pretty much the same way; in what follows the structure is described in some detail.

The monoidal structure is given by additive parallel composition $\square^{T \text {-Res }}$ of resumptions, which is derived from $\square$ on components (Section 4.2), the corresponding outer structure. It acts on objects as sum of sets:

$$
A \square^{T-\operatorname{Res}} B:=A+B .
$$

On arrows, its action

$$
\begin{aligned}
\square_{A, C, B, D}^{T \text {-Res }}: Z_{A, B} \times Z_{C, D} \longrightarrow Z_{A+C, B+D}, \quad \text { that is } \\
\operatorname{Hom}_{T \text {-Res }}(A, B) \times \operatorname{Hom}_{T \text {-Res }}(C, D) \longrightarrow \operatorname{Hom}_{T-\text { Res }}(A+C, B+D)
\end{aligned}
$$

is induced via the following diagram similar to $(22)$.

$$
\begin{aligned}
& F_{A+C, B+D}\left(Z_{A, B} \times Z_{C, D}\right)-\cdots-\rightarrow F_{A+C, B+D}\left(Z_{A+C, B+D}\right) \\
& \zeta_{A, B} \square \zeta_{C, D} \uparrow \quad \text { final } \uparrow \zeta_{A+C, B+D}
\end{aligned}
$$

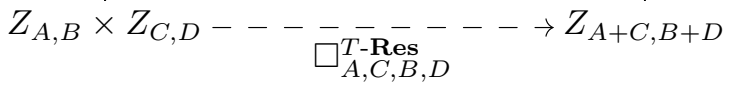

Lemma 7.7. The mapping $\square^{T \text {-Res }}$ yields a functor $\square^{T \text {-Res }}: T$-Res $\times T$-Res $\rightarrow T$-Res.

Proof. Let us first prove preservation of identities. We have the following successive morphisms of coalgebraic components, where coalgebras $X \rightarrow F X$ are written horizontally.

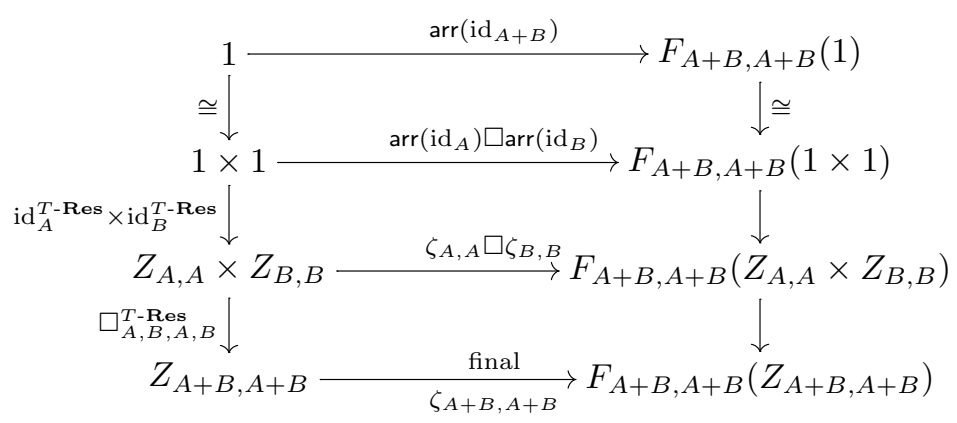

The first square commutes because of the compatibility of $\square$ and arr (Lemma 4.9.1); the second does because of the definition of $\mathrm{id}^{T \text {-Res }}(23)$ and functoriality of $\square$ (right after Definition 4.7); and the third one is the definition of $\square^{T \text {-Res }}(25)$. This proves that the following diagram commutes, since the two composites are both morphisms from the component $\operatorname{arr}\left(\operatorname{id}_{A+B}\right)$ to the final $\zeta_{A+B, A+B}$.

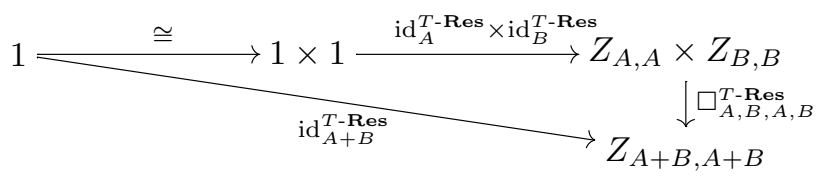

Hence $\square^{T \text {-Res }}: T$-Res $\times T$-Res $\rightarrow T$-Res preserves identities. 
We turn to preservation of composition; the arguments proceed in a similar way. We have the following two (parallel) series of morphisms of coalgebraic components. They are all arrows in the category $\operatorname{Comp}\left(T, A+B, A^{\prime \prime}+B^{\prime \prime}\right)$.

$$
\begin{aligned}
& \left(\zeta_{A, A^{\prime}} \gg \zeta_{A^{\prime}, A^{\prime \prime}}\right) \square\left(\zeta_{B, B^{\prime}} \gg \zeta_{B^{\prime}, B^{\prime \prime}}\right) \\
& \begin{array}{c}
\stackrel{\cong}{\stackrel{\left(\square_{A, B, A^{\prime}, B^{\prime}}^{T-\text { Res }}\right) \times\left(\square_{A^{\prime}, B^{\prime}, A^{\prime \prime}, B^{\prime \prime}}^{T-\text { Res }}\right)}{\longrightarrow}} \longrightarrow \zeta_{A+B, A^{\prime}+B^{\prime}} \gg \zeta_{A^{\prime}+B^{\prime}, A^{\prime \prime}+B^{\prime \prime}} \\
\stackrel{\mathrm{o}_{A+B, A^{\prime}+B^{\prime}, A^{\prime \prime}+B^{\prime \prime}}^{T-\text { Res }}}{\longrightarrow} \zeta_{A+B, A^{\prime \prime}+B^{\prime \prime}} ;
\end{array} \\
& \begin{aligned}
\stackrel{\left.\mathrm{o}_{A, A^{\prime}, A^{\prime \prime}}^{T-\text { Res }}\right) \times\left(\mathrm{o}_{B, B^{\prime}, B^{\prime \prime}}^{T-\text { Res }}\right)}{{ }^{T}} & \left(\zeta_{A, A^{\prime}} \gg \zeta_{A^{\prime}, A^{\prime \prime}}\right) \square\left(\zeta_{B, B^{\prime}} \gg \zeta_{B^{\prime}, B^{\prime \prime}}\right) \\
\hline \square_{A, B, A^{\prime \prime}, B^{\prime \prime}}^{T-\text { Res }} & \zeta_{A, A^{\prime \prime}} \square \zeta_{B, B^{\prime \prime}} \\
\longrightarrow & \zeta_{A+B, A^{\prime \prime}+B^{\prime \prime} .}
\end{aligned}
\end{aligned}
$$

The first isomorphism is due to Lemma 4.9.2; the second one is a morphism of components due to the definition of $\square^{T \text {-Res }}$ and functoriality of $\gg$ (Lemma 4.2.4); and the third one is the definition of $o^{T-R e s}$. The other two are component morphisms for similar reasons. Since the coalgebraic component $\zeta_{A+B, A^{\prime \prime}+B^{\prime \prime}}$ is a final coalgebra, we conclude that the two composites above are identical. In particular, by taking their underlying functions, we have a commuting diagram

$$
\begin{aligned}
& \left(Z_{A, A^{\prime}} \times Z_{A^{\prime}, A^{\prime \prime}}\right) \times\left(Z_{B, B^{\prime}} \times Z_{B^{\prime}, B^{\prime \prime}}\right) \cong\left(Z_{A, A^{\prime}} \times Z_{B, B^{\prime}}\right) \times\left(Z_{A^{\prime}, A^{\prime \prime}} \times Z_{B^{\prime}, B^{\prime \prime}}\right)
\end{aligned}
$$

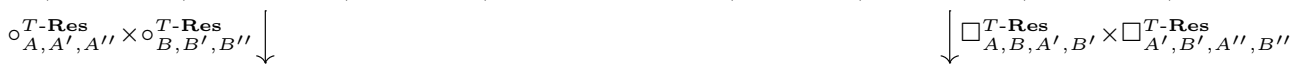

$$
\begin{aligned}
& Z_{A, A^{\prime \prime}} \times Z_{B, B^{\prime \prime}} \quad Z_{A+B, A^{\prime}+B^{\prime}} \times Z_{A^{\prime}+B^{\prime}, A^{\prime \prime}+B^{\prime \prime}} \\
& \square_{A, B, A^{\prime \prime}, B^{\prime \prime}}^{T-\text { Res }} Z_{A+B, A^{\prime \prime}+B^{\prime \prime}} \overbrace{A+B, A^{\prime}+B^{\prime}, A^{\prime \prime}+B^{\prime \prime}}^{0^{T-R e s}}
\end{aligned}
$$

in Set. Hence $\square^{T \text {-Res }}: T$-Res $\times T$-Res $\rightarrow T$-Res preserves composition of arrows.

The monoidal unit for ( $T$-Res, $\square^{T-\mathbf{R e s}}$ ) is the empty set 0 . It remains to describe associativity, unit and symmetry isomorphisms; they appear in the proof of the following result.

Proposition 7.8. ( $T$-Res, $\square^{T \text {-Res }}, 0$ ) is a symmetric monoidal category.

Proof. We shall first describe the definition of the structural isomorphisms. Then we prove that: 1) they are indeed isomorphisms; 2) they are natural; and 3) they are coherent as in (Mac Lane, 1998).

An associativity isomorphism $\alpha^{T \text {-Res }}: A+(B+C) \cong(A+B)+C$ in $T$-Res-recall that $\square^{T \text {-Res }}$ is + on objects, see $(24)$-is induced by the following diagram.

$$
\begin{array}{r}
F_{A+(B+C),(A+B)+C}(1)---\rightarrow F_{A+(B+C),(A+B)+C}\left(Z_{A+(B+C),(A+B)+C}\right) \\
\operatorname{arr}\left(\alpha_{+}\right) \uparrow \\
\text { final } \uparrow \zeta_{A+(B+C),(A+B)+C} \\
1-----\overline{\alpha_{A, B, C}^{T-\overline{R e s}}}---\rightarrow Z_{A+(B+C),(A+B)+C}
\end{array}
$$

Here $\alpha_{+}$on the left, in $\operatorname{arr}\left(\alpha_{+}\right)$, is the isomorphism $A+(B+C) \cong(A+B)+C$ in Set. 
In exactly the same way we obtain unit isomorphisms $\lambda^{T \text {-Res }}, \rho^{T \text {-Res }}$ and symmetry isomorphisms $\gamma^{T \text {-Res }}$, from the corresponding isomorphisms for + in Set.

It is easy to see that all these are indeed isomorphisms. Let us write down the proof for $\alpha^{T \text {-Res }}$. Let $\bar{\alpha}_{A, B, C}^{T \text {-Res }}$ be the following resumption induced by the isomorphism $\alpha_{+}^{-1}$ : $(A+B)+C \stackrel{\cong}{\longrightarrow} A+(B+C)$ in Set:

$$
\begin{array}{r}
F_{(A+B)+C, A+(B+C)}(1)---\rightarrow F_{(A+B)+C, A+(B+C)}\left(Z_{(A+B)+C, A+(B+C)}\right) \\
\operatorname{arr}\left(\alpha_{+}^{-1}\right) \uparrow \\
\quad \text { final } \uparrow \zeta_{(A+B)+C, A+(B+C)}
\end{array}
$$

We claim that this $\bar{\alpha}_{A, B, C}^{T \text {-Res }}$ is the inverse of $\alpha_{A, B, C}^{T \text {-Res }}$, that is,

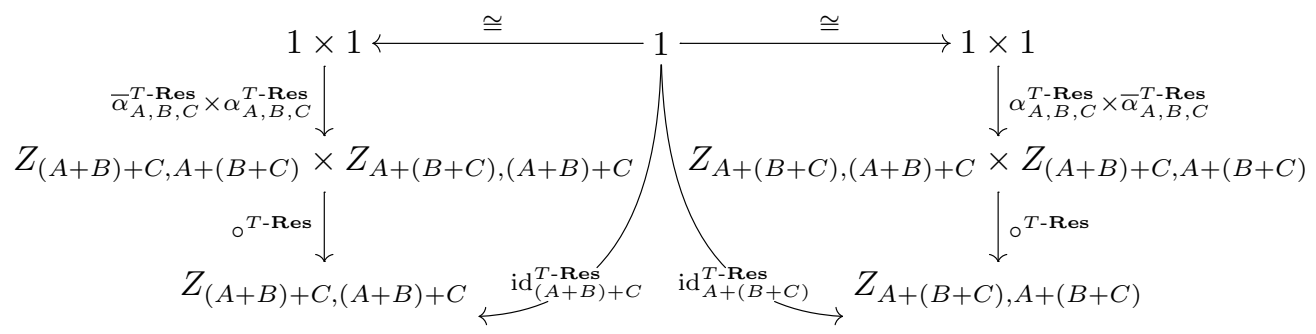

We prove commutativity of the triangle on the right; the other is similar. As before, this is by proving that the following arrows are all component morphisms leading to a final coalgebra $\zeta_{A+(B+C), A+(B+C)}$.

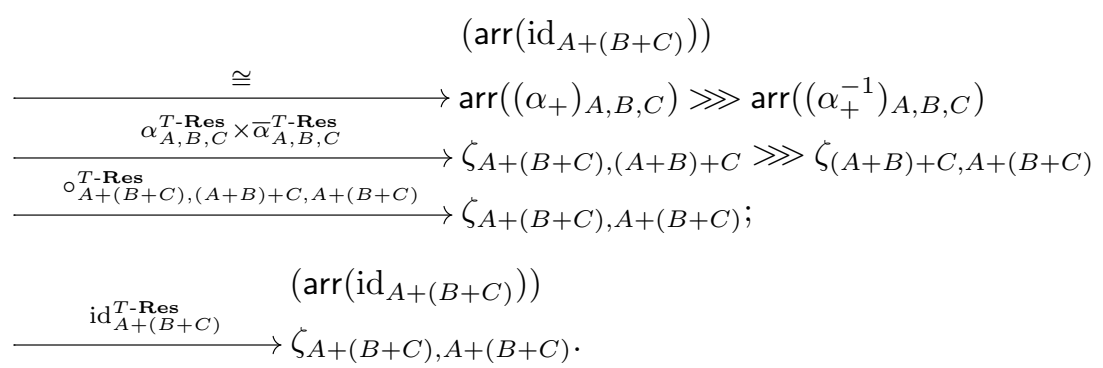

The first isomorphism is because of Lemma 4.2.1; the second one is due to the definition of $\alpha^{T \text {-Res }}, \bar{\alpha}^{T \text {-Res }}$ and functoriality of $\gg$ (Lemma 4.2.4); the third one is the definition of $\circ^{T-R e s}$; and the last is the definition of $\mathrm{id}^{T-\text { Res }}$. Thus we have proved that $\alpha_{A, B, C}^{T-\text { Res }}$ is indeed an isomorphism.

We turn to the naturality of $\alpha^{T-\text { Res }}, \lambda^{T \text {-Res }}, \rho^{T \text {-Res }}$ and $\gamma^{T \text {-Res }}$; again we present only the proof for $\alpha^{T \text {-Res }}$. It means commutativity of

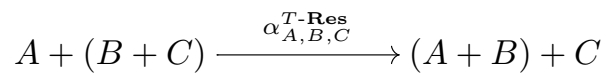

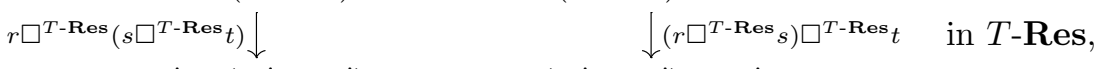

$$
\begin{aligned}
& A^{\prime}+\left(B^{\prime}+C^{\prime}\right) \underset{\alpha_{A^{\prime}, B^{\prime}, C^{\prime}}^{T-\text { Res }}}{\longrightarrow}\left(A^{\prime}+B^{\prime}\right)+C^{\prime}
\end{aligned}
$$


for any resumptions $r, s$ and $t$ of suitable types. This amounts to, in Set

$$
\begin{aligned}
& 1 \times\left(\left(Z_{A, A^{\prime}} \times Z_{B, B^{\prime}}\right) \times Z_{C, C^{\prime}}\right) \longrightarrow\left(Z_{A, A^{\prime}} \times\left(Z_{B, B^{\prime}} \times Z_{C, C^{\prime}}\right)\right) \times 1 \\
& \begin{array}{cc}
\alpha_{A, B, C}^{T-\text { Res }} \times\left(\square^{T-\text {Res}} \circ\left(\square^{T-\text { Res }} \times Z_{C, C^{\prime}}\right)\right) & \mid \\
\downarrow & \left(\square^{T-\text { Res }} \circ\left(Z_{A, A^{\prime}} \times \square^{T-\text { Res }}\right)\right) \times \alpha_{A^{\prime}, B^{\prime}, C^{\prime}}^{T-\text { Res }} \\
\downarrow & \downarrow
\end{array} \\
& Z_{A+(B+C),(A+B)+C} \quad Z_{A+(B+C), A^{\prime}+\left(B^{\prime}+C^{\prime}\right)} \\
& \times Z_{(A+B)+C,\left(A^{\prime}+B^{\prime}\right)+C^{\prime}} \times Z_{A^{\prime}+\left(B^{\prime}+C^{\prime}\right),\left(A^{\prime}+B^{\prime}\right)+C^{\prime}} \\
& \overbrace{A+(B+C),\left(A^{\prime}+B^{\prime}\right)+C^{\prime}}^{Z^{T-\text { Res }}}
\end{aligned}
$$

Once again, this is by showing that the two above composites are parallel coalgebra morphisms leading to a final coalgebra. Namely

$$
\begin{aligned}
& \operatorname{arr}\left(\left(\alpha_{+}\right)_{A, B, C}\right) \gg\left(\left(\zeta_{A, A^{\prime}} \square \zeta_{B, B^{\prime}}\right) \square \zeta_{C, C^{\prime}}\right) \\
& \begin{array}{c}
\stackrel{\cong}{\stackrel{\left(\square^{T-\text { Res }} \circ\left(Z_{A, A^{\prime}} \times \square^{T-\text { Res }}\right)\right) \times \alpha_{A^{\prime}, B^{\prime}, C^{\prime}}^{T-\text { Res }}}{\longrightarrow}}\left(\zeta_{A+(B+C), A^{\prime}+\left(B^{\prime}+C^{\prime}\right)} \gg \zeta_{A^{\prime}+\left(B^{\prime}+C^{\prime}\right),\left(A^{\prime}+B^{\prime}\right)+C^{\prime}}\right. \\
\stackrel{\mathrm{o}_{A+(B+C),\left(A^{\prime}+B^{\prime}\right)+C^{\prime}, A^{\prime}+\left(B^{\prime}+C^{\prime}\right)}^{T-\text { Res }}}{\longrightarrow} \zeta_{A+(B+C),\left(A^{\prime}+B^{\prime}\right)+C^{\prime}}
\end{array}
\end{aligned}
$$

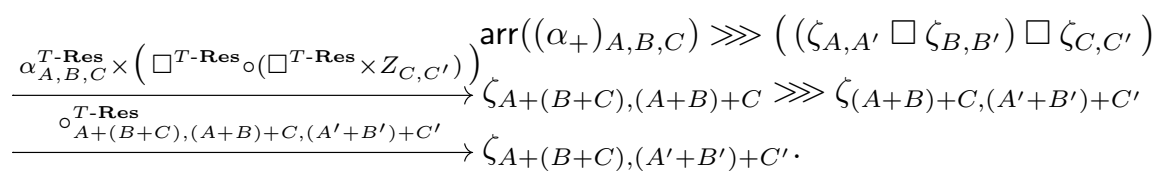

The first one is an isomorphism of components due to Lemma 4.9.3; the others are similar to the ones earlier in this section. This concludes the proof of naturality of $\alpha^{T-\text { Res }}$.

Finally, we need to check the standard coherence conditions for $\alpha^{T \text {-Res }}, \lambda^{T \text {-Res }}, \rho^{T \text {-Res }}$ and $\gamma^{T \text {-Res }}$ in a symmetric monoidal category, as described in e.g. (Mac Lane, 1998). Let us prove

$$
A+(0+B) \stackrel{\alpha_{A, 0, B}^{T-\text { Res }}}{\cong} \underset{\operatorname{id}_{A}^{T-\operatorname{Res}} \square^{T-\text { Res }} \lambda_{B}^{T-\text { Res }} \longrightarrow}{\cong}(A+B)+B \quad \text { in T-Res; }
$$

the others are similar. The above diagram amounts to the following diagram in Set.

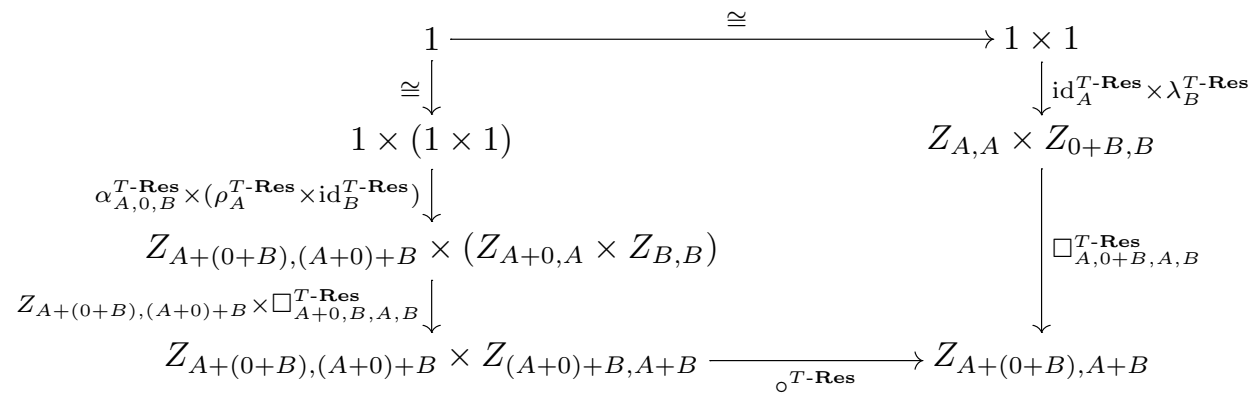


Yet once again, this diagram commutes because the two composites are (parallel) coalgebra morphisms from the coalgebra

$$
\begin{array}{r}
\operatorname{arr}\left[A+(0+B) \stackrel{A+\lambda_{+}}{\longrightarrow} A+B\right] \stackrel{(*)}{=} \operatorname{arr}\left[A+(0+B) \stackrel{\alpha_{+}}{\longrightarrow}(A+0)+B \stackrel{\rho_{++} B}{\longrightarrow} A+B\right] \\
: 1 \longrightarrow F_{A+(0+B), A+B}(1)
\end{array}
$$

to the final $\zeta_{A+(0+B), A+B}$. The equality $(*)$ is due to the same coherence condition as $(26)$ for the monoidal category (Set,,+ 0$)$; the other details can be easily filled in. This concludes the proof.

\subsection{Trace structure for resumptions}

A trace/feedback operator $\operatorname{Tr}^{T-\text { Res }}$ for resumptions is induced by the "outer" operator $\operatorname{Tr}$ for components; this is exactly in the same way as we derived e.g. the tensor $\square^{T \text {-Res }}$ from the outer $\square$. Let us nevertheless spell out how it is done concretely.

For arbitrary sets $A, B, C \in T$-Res, the trace operator

$$
\begin{aligned}
& \operatorname{Tr}_{A, B, C}^{T \text {-Res }}: Z_{A+C, B+C} \longrightarrow Z_{A, B}, \quad \text { that is } \\
& \qquad \operatorname{Hom}_{T-\operatorname{Res}}(A+C, B+C) \longrightarrow \operatorname{Hom}_{T-\operatorname{Res}}(A, B)
\end{aligned}
$$

is introduced by the following coinduction diagram:

$$
\begin{aligned}
& F_{A, B}\left(Z_{A+C, B+C}\right)----\rightarrow F_{A, B}\left(Z_{A, B}\right) \\
& \operatorname{Tr}\left(\zeta_{A+C, B+C}\right) \uparrow \quad \text { final } \uparrow \zeta_{A, B} \\
& Z_{A+C, B+C}--\overline{\operatorname{Tr}_{A, B, C}^{T}-\overline{\text { Res }}}-\rightarrow Z_{A, B}
\end{aligned}
$$

Here the operator $\operatorname{Tr}$ on the left, acting on $\zeta_{A+C, B+C}$, is the trace operator for components from Definition 6.1.

It is again straightforward to prove that $\operatorname{Tr}^{T \text {-Res }}$ satisfies the trace axioms: each axiom is essentially reduced to the corresponding axiom on $\operatorname{Tr}$ for components. Let us prove the post-composition/tightening axiom (21) for example. It amounts to the following commutative diagram.

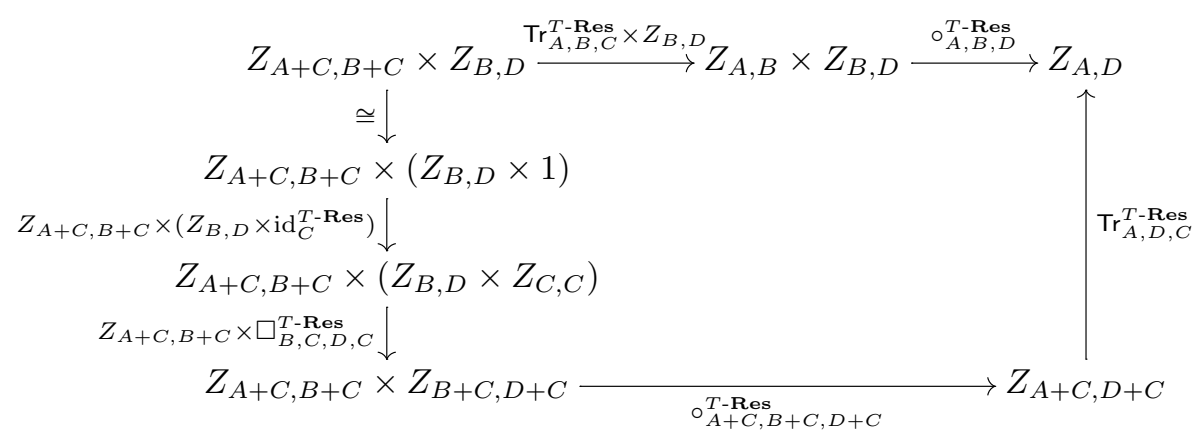


The composite on the top row is a coalgebra morphism

$$
\begin{aligned}
& \operatorname{Tr}\left(\zeta_{A+C, B+C}\right) \gg \zeta_{B, D} \\
\stackrel{\operatorname{Tr}_{A, B, C}^{T-\text { Res }} \times Z_{B, D}}{\longrightarrow} & \zeta_{A, B} \gg \zeta_{B, D} \\
\stackrel{0_{A, B, D}^{T-\text { Res }}}{\longrightarrow} & \zeta_{A, D} ;
\end{aligned}
$$

where the first arrow is a coalgebra morphism due to the definition of $\operatorname{Tr}^{T-R e s}$ and functoriality of $\gg$ (Lemma 4.2.4); the latter is due to the definition of $\circ^{T-R e s}$.

The other composite in (27) is a morphism between the same coalgebras:

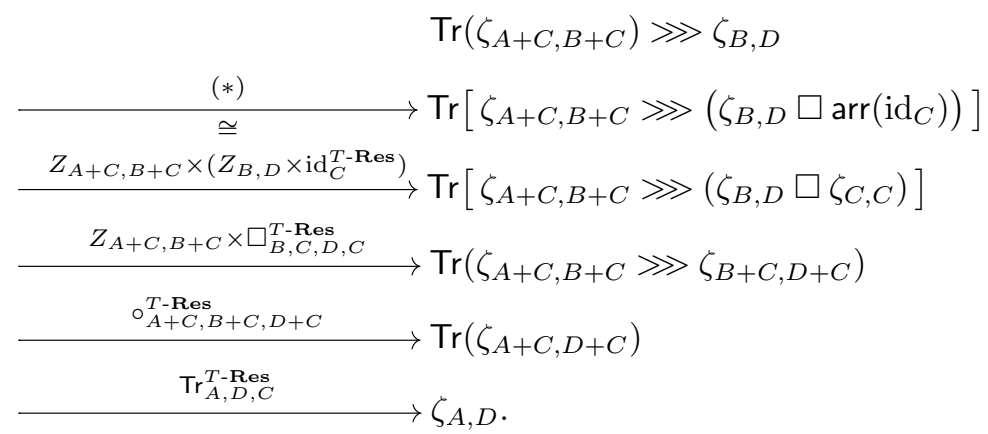

Here the isomorphism $(*)$ is due to the post-composition naturality for Tr, see Section 6.3. The last morphism is the definition of $\operatorname{Tr}^{T-\text { Res }}$. The other arrows are also component morphisms; here the functoriality of $\operatorname{Tr}$ (Lemma 6.2) is crucial. Recall that the functor $\operatorname{Tr}: \operatorname{Comp}(T, A+C, B+C) \rightarrow \operatorname{Comp}(T, A, B)$ acts on arrows as the identity. We conclude, by the finality of $\zeta_{A, D}$, that the diagram (27) commute.

The other axioms can be verified in the same manner. This concludes the proof of Theorem 7.4.

\section{Concluding remarks}

This paper is part of an ongoing line of research into the mathematical (coalgebraic) foundations of components as basic building blocks in computing (see (Szyperski, 1998) for a wider perspective). Obviously, connections to existing component languages like Reo (Arbab, 2004; Baier et al., 2006) need to be explored. There are also several directions for further, more mathematically oriented, research about coalgebraic components. We briefly mention two such avenues, involving duality and dynamic logic.

For specific monads, like for powerset on the category of sets or for the identity on the category of Hilbert spaces, the associated Kleisli category carries a dagger operator $\dagger$ that commutes with the tensor and biproduct. Via this dagger one can define a duality operator:

$$
\begin{gathered}
\operatorname{Comp}(T, A, B)^{\mathrm{op}} \stackrel{(-)^{\star}}{\longrightarrow} \operatorname{Comp}(T, B, A) \\
(X \otimes A \stackrel{c}{\rightarrow} X \otimes B) \longmapsto\left(X \otimes B \stackrel{c^{\dagger}}{\rightarrow} X \otimes A\right)
\end{gathered}
$$

It satisfies, for instance $(c \| d)^{\star}=\left(c^{\star} \| d^{\star}\right)$. Such a duality introduces a form of re- 
versible computation that may be useful in capturing aspects of quantum computing coalgebraically, see also (Abramsky, 2009).

Another interesting topic for further research is how to extend modal logic for coalgebras to a dynamic logic (see e.g. (Goldblatt, 1992)) for coalgebraic components. In such a logic one expects basic compositionality properties (see also (Klin, 2009)), so that for instance $\square_{c \gg d}, \square_{c} \square d$ etc. can be expressed in terms of $\square_{c}$ and $\square_{d}$.

\section{Acknowledgements}

Thanks are due to Masahito Hasegawa, Chris Heunen and Ana Sokolova for fruitful discussions; to Micah Blake McCurdy for his inspiring work; to Shin-ya Katsumata for pointing out the relevance of Micah's work; and to two anonymous referees for their useful comments.

\section{References}

Abramsky, S. (2009). Coalgebras, Chu spaces, and representations of physical systems. http: //arxiv.org/0910.3959.

Abramsky, S., Haghverdi, E., and Scott, P. (2002). Geometry of interaction and linear combinatory algebras. Math. Struct. in Comp. Sci., 12(5):625-665.

Abramsky, S. and Jagadeesan, R. (1994). Games and full completeness for multiplicative linear logic. Journ. Symb. Logic, 59(2):543-574.

Arbab, F. (2004). Reo: a channel-based coordination model for component composition. Math. Struct. in Comp. Sci., 14(3):329-366.

Asada, K. and Hasuo, I. (2010). Categorifying computations into components via arrows as profunctors. In Jacobs, B., Niqui, M., Rutten, J., and Silva, A., editors, Coalgebraic Methods in Computer Science (CMCS 2010), volume 264 of Elect. Notes in Theor. Comp. Sci., pages $25-45$.

Baez, J. C. and Dolan, J. (1998). Higher dimensional algebra III: $n$-categories and the algebra of opetopes. Adv. Math, 135:145-206.

Baier, C., Sirjani, M., Arbab, F., and Rutten, J. J. M. M. (2006). Modeling component connectors in Reo by constraint automata. Science of Comput. Progr., 61(2):75-113.

Barbosa, L. (2001). Components as Coalgebras. PhD thesis, Univ. Minho.

Barbosa, L. S. (2003). Towards a calculus of state-based software components. Journ. of Universal Comp. Sci., 9(8):891-909.

Barr, M. (1993). Terminal coalgebras in well-founded set theory. Theor. Comp. Sci., 114(2):299315. Corrigendum in Theor. Comp. Sci. 124:189-192, 1994.

Borceux, F. (1994). Handbook of Categorical Algebra, volume 50, 51 and 52 of Encyclopedia of Mathematics. Cambridge Univ. Press.

Cockett, J. R. B. and Seely, R. A. G. (1999). Linearly distributive functors. Journ. of Pure EG Appl. Algebra, 143(1-3):155-203.

Goldblatt, R. (1992). Logics of Time and Computation. CSLI Lecture Notes 7, Stanford, $2^{\text {nd }}$ rev. edition.

Haghverdi, E. (2000). A Categorical Approach to Linear Logic, Geometry of Proofs and Full Completeness. PhD thesis, Univ. of Ottawa.

Hasegawa, M. (1999). Models of Sharing Graphs : A Categorical Semantics of Let and Letrec. Distinguished Dissertations in Computer Science. Cambridge Univ. Press. 
Hasegawa, M. (2004). The uniformity principle on traced monoidal categories. Publ. RIMS, Kyoto Univ., 40(3):991-1014.

Hasuo, I., Heunen, C., Jacobs, B., and Sokolova, A. (2009). Coalgebraic components in a manysorted microcosm. In Kurz, A. and Tarlecki, A., editors, Conference on Algebra and Coalgebra in Computer Science (CALCO 2009), number 5728 in Lect. Notes Comp. Sci., pages 64-80. Springer, Berlin.

Hasuo, I., Jacobs, B., and Sokolova, A. (2007). Generic trace semantics via coinduction. Logical Methods in Comp. Sci., 3(4:11).

Hasuo, I., Jacobs, B., and Sokolova, A. (2008). The microcosm principle and concurrency in coalgebra. In Foundations of Software Science and Computation Structures, volume 4962 of Lect. Notes Comp. Sci., pages 246-260. Springer-Verlag.

Hughes, J. (2000). Generalising monads to arrows. Science of Comput. Progr., 37(1-3):67-111.

Jacobs, B. (1999). Categorical Logic and Type Theory. North Holland, Amsterdam.

Jacobs, B. (2010). From coalgebraic to monoidal traces. In Jacobs, B., Niqui, M., Rutten, J., and Silva, A., editors, Coalgebraic Methods in Computer Science (CMCS 2010), volume 264 of Elect. Notes in Theor. Comp. Sci., pages 125-140. Elsevier.

Jacobs, B., Heunen, C., and Hasuo, I. (2009). Categorical semantics for arrows. Journ. Funct. Progr., 19(3-4):403-438.

Jacobs, B. and Rutten, J. J. M. M. (1997). A tutorial on (co)algebras and (co)induction. EATCS Bulletin, 62:222-259.

Janelidze, G. and Kelly, M. (2001). A note on actions of a monoidal category. Theory and Applications of Categories, 9(4):61-91. www.tac.mta.ca/tac/volumes/9/n4/9-04abs.html.

Joyal, A. and Street, R. (1991). The geometry of tensor calculus, I. Adv. Math, 88:55-112.

Joyal, A., Street, R., and Verity, D. (1996). Traced monoidal categories. Math. Proc. Cambridge Phil. Soc., 119(3):425-446.

Katis, P., Sabadini, N., and Walters, R. F. C. (1997). Bicategories of processes. Journ. of Pure \& Appl. Algebra, 115(2):141-178.

Kelly, G. M. and Street, R. (1974). Review of the elements of 2-categories. In Kelly, G. M., editor, Proc. Sydney Category Theory Seminar 1972/1973, number 420 in Lect. Notes Math., pages 75-103. Springer, Berlin.

Klin, B. (2009). Bialgebraic methods and modal logic in structural operational semantics. Inf. Comput., 207(2):237-257.

Krstić, S., Launchbury, J., and Pavlović, D. (2001). Categories of processes enriched in final coalgebras. In Honsell, F. and Miculan, M., editors, Foundations of Software Science and Computation Structures, number 2030 in Lect. Notes Comp. Sci., pages 303-317. Springer, Berlin.

Kurz, A. and Pattinson, D. (2005). Coalgebraic modal logic of finite rank. Math. Struct. in Comp. Sci., 15(3):453-473.

Mac Lane, S. (1998). Categories for the Working Mathematician. Springer, Berlin, 2nd edition.

McCurdy, M. B. (2010). The Tannaka-representation adjunction for weak bialgebras and separable Frobenius monoidal functors. Forthcoming $\mathrm{PhD}$ thesis, Macquarie University.

Melliès, P.-A. (2006). Functorial boxes in string diagrams. In Ésik, Z., editor, CSL, volume 4207 of Lecture Notes in Computer Science, pages 1-30. Springer.

Milner, R. (1975). Processes: a mathematical model of computing agents. In Logic Colloq. 'ry, pages 157-173. North-Holland.

Milner, R. (1980). A Calculus of Communicating Systems. Lect. Notes Comp. Sci. Springer, Berlin.

Moggi, E. (1991). Notions of computation and monads. Inf. \& Comp., 93(1):55-92. 
Pattinson, D. (2003). An introduction to the theory of coalgebras. Course notes for NASSLLI. www. indiana.edu/ ${ }^{\sim}$ nasslli.

Penrose, R. (1971). Applications of negative dimensional tensors. In Welsh, D., editor, Combinatorial Mathematics and its Applications, pages 221-244. Academic Press.

Rutten, J. J. M. M. (2000). Universal coalgebra: a theory of systems. Theor. Comp. Sci., 249:3-80.

Rutten, J. J. M. M. (2006). Algebraic specification and coalgebraic synthesis of Mealy automata. Elect. Notes in Theor. Comp. Sci., 160:305-319.

Selinger, P. (2010). A survey of graphical languages for monoidal categories. In Coecke, B., editor, New Structures for Physics, Lect. Notes Phys. Springer-Verlag.

Simpson, A. K. and Plotkin, G. D. (2000). Complete axioms for categorical fixed-point operators. In Logic in Computer Science, pages 30-41.

Sokolova, A. (2005). Coalgebraic Analysis of Probabilistic Systems. PhD thesis, Techn. Univ. Eindhoven.

Szyperski, C. (1998). Component Software. Addison-Wesley.

Turi, D. and Plotkin, G. (1997). Towards a mathematical operational semantics. In Logic in Computer Science, pages 280-291. IEEE, Computer Science Press. 